\title{
Thermal fluctuations and bending rigidity of bilayer membranes
}

\author{
Pedro Tarazona, ${ }^{1, a)}$ Enrique Chacón, ${ }^{2, b)}$ and Fernando Bresme ${ }^{3, c)}$ \\ ${ }^{1}$ Departamento de Física Teórica de la Materia Condensada, Condensed Matter Physics Center (IFIMAC) and \\ Instituto de Ciencia de Materiales Nicolás Cabrera, Universidad Autónoma de Madrid, Madrid 28049, Spain \\ ${ }^{2}$ Instituto de Ciencia de Materiales de Madrid, CSIC, 28049 Madrid, Spain and Instituto de Ciencia de \\ Materiales Nicolás Cabrera, Universidad Autónoma de Madrid, Madrid 28049, Spain \\ ${ }^{3}$ Department of Chemistry, Imperial College London, SW7 2AZ London, United Kingdom and Department \\ of Chemistry, Norwegian University of Science and Technology, Trondheim, Norway
}

(Received 20 June 2013; accepted 31 July 2013; published online 5 September 2013)

\begin{abstract}
We present a new scheme to estimate the elastic properties of biological membranes in computer simulations. The method analyzes the thermal fluctuations in terms of a coupled undulatory mode, which disentangle the mixing of the mesoscopic undulations and the high-q protrusions. This approach makes possible the accurate estimation of the bending modulus both for membranes under stress and in tensionless conditions; it also extends the applicability of the fluctuation analysis to the small membrane areas normally used in atomistic simulations. Also we clarify the difference between the surface tension imposed in simulations through a pressure coupling barostat, and the surface tension that can be extracted from the analysis of the low wave vector dependence of the coupled undulatory fluctuation spectrum. The physical analysis of the peristaltic mode is also refined, by separating the bulk and protrusions contributions. We illustrate the procedure by analyzing 1-palmitoyl-2-oleoylsn-glycero-3-phosphocholine and 1,2-dipalmitoyl-sn-glycero-3-phosphocholine bilayers. The bending moduli obtained from our analysis, shows good agreement with available experiments. (C) 2013 AIP Publishing LLC. [http://dx.doi.org/10.1063/1.4818421]
\end{abstract}

\section{INTRODUCTION}

Lipid bilayers are the main structural components of biological membranes. Their elastic properties play a key role in determining the anchoring, insertion, and function of transmembrane proteins. ${ }^{1}$ Hence, there is considerable interest in understanding the factors that regulate the mechanical properties of membranes, and how the presence of solutes affects these properties. ${ }^{2}$ A quantitative knowledge of the membrane elasticity is also necessary to explain and predict the formation of transient structures in membrane fusion processes. ${ }^{3}$ We report in our paper a new computational approach to quantify the membrane elastic properties from the analysis of the membrane fluctuation modes.

The macroscopic-mesoscopic fluctuation behavior of membranes can be well described by Helfrich effective surface Hamiltonian, ${ }^{4}$ which models the membrane as a geometrical surface, neglecting all the molecular details of its structure. Within that description, the elastic properties are determined by experimentally measurable thermodynamic parameters, namely, the surface tension and the bending modulus of fluid membranes. This formalism has significantly advanced our understanding of the geometrical shapes adopted by membranes as well as the entropy associated to the membrane fluctuations. ${ }^{4,5}$ Experimental and computer simulation data ${ }^{2,6,7}$ are often fitted to the Helfrich Hamiltonian, as an approach to extract the bending modulus and its dependence with the chemical composition of the lipid bilayer. Despite the

\footnotetext{
a)Electronic mail: pedro.tarazona@uam.es

b) Electronic mail: echacon@icmm.csic.es

c)Electronic mail: f.bresme@imperial.ac.uk
}

success of this approach, there are many important aspects of a bilayer membrane that are obviously beyond a mesoscopic description, in particular, the fluctuations of the local thickness of the membrane or the protrusions of the lipid heads out of the bilayer plane. The characterization of these microscopic degrees of freedom, beyond the mesoscopic description given by the Helfrich Hamiltonian, may provide relevant information to understand the complexes formed by proteins at, and across, bilayer membranes. In this respect, molecular simulations offer one of the most powerful microscopic approaches to extract information on the nature of these nonmesoscopic fluctuations. We note that simplified theoretical models, ${ }^{8-11}$ as well as Ginzburg-Landau approaches, ${ }^{12}$ have allowed some progress in the analysis of these fluctuations, but we believe that only through computer simulations of more detailed models it is possible to gain accurate information on the intramolecular and intermolecular correlations.

The characterization of the collective elastic properties of bilayer membranes from computer simulations has been actively investigated over the last decades. An uncertainty associated to that analysis comes from the difficulty in setting a specific length scale that separates the range of mesoscopic and microscopic (molecular) fluctuations undergone by the membranes. This problem has prompted discussions on the equivalence between the bending modulus associated to the thermal fluctuations and the bending modulus defining the equilibrium shape of cylindrical surfaces. A controversy that has been recently solved ${ }^{7}$ using computer simulations of cylindrical lipid bilayers. These simulations have shown excellent agreement between the bending modulus extracted from the active deformation of the membrane and that 
obtained from the analysis of the thermal fluctuations. The active deformation technique is a usual way to quantify the elastic constants in the context of density functional calculations of model of membranes, ${ }^{13}$ however, this approach is very difficult to implement in numerical simulations. A new active deformation procedure to evaluate $\kappa$ has been recently reported. The method relies on the measurement of the force needed to buckle a membrane. ${ }^{14}$ In addition to the bending modulus equivalence, another open question is whether the surface tension obtained from the analysis of the thermal fluctuation spectrum of the flat membrane, agrees ${ }^{15-17}$ or differs ${ }^{18-20}$ from the externally imposed tension. This is a question we clarify in the present paper.

Over the last two decades, the fluctuations of bilayer membranes have been described as a combination of undulatory and peristaltic modes, both for simplified ${ }^{21,22}$ and realistic models of membranes. ${ }^{6,23}$ In our view, the main problem with current approaches is connected to the description of the undulatory mode, which exhibits a crossover between the coupled regime, in which the bilayer fluctuates as a whole, and the uncoupled fluctuation of each layer, which is usually referred to as protrusion. ${ }^{24}$ The transition between these two modes is smooth, going from the fully coupled mode, $q$ $\ll 2 \pi / d$, to the fully uncoupled protrusions, $q \gtrsim 2 \pi / d$, where $q$ is the wavevector that quantifies the fluctuation modes, and $d$ the mean distance between the membrane monolayers. The fluctuations in these two regimes are very different, making difficult to derive a simple analytical dependence that fits the mean square amplitude of the undulatory mode over the whole $q$ range. Several expressions ${ }^{6,23}$ have been proposed to model the fluctuations in the small and large$q$ regimes. Some approaches describe the membrane elasticity in terms of model interactions between its components, in order to obtain a single equation to cover the full range of undulatory and peristaltic fluctuations. A theory of coupled elastic monolayers ${ }^{25,26}$ has been used to describe accurately both tensionless membranes ${ }^{27}$ and membranes under tension. ${ }^{22}$ The effects of the molecular tilt have also been introduced. ${ }^{28,29}$ These studies claim that the phospholipid tilt may be more relevant than the protrusions. Using this approach it was shown that the simulation data can be very well fitted, even for membrane lateral lengths of a few nanometers. ${ }^{30}$ Based on this procedure a very interesting alternative method to study the bending modulus from simulations of modest size has been published, ${ }^{31}$ in which the authors obtained the bending modulus from the analysis of the fluctuations of predefined order parameters that define the orientation of the lipids. Watson et al. approach and the approach presented here, include pure undulatory modes and protrusion modes by construction. To achieve the separation between these two modes, the protrusion modes observed in the fluctuations of the mean surface (undulatory mode), are filtered by sampling specific correlation across the membrane. In our case, we choose the correlation between the surfaces defined by the head groups of the two phospholipid layers, while in the work of Watson $e t a l{ }^{31}$ they use the local orientation of the aliphatic chains. Very recently, an alternative description of the undulatory spectrum of the tensionless membranes has been proposed, ${ }^{32}$ including the static number density structure factor of the monolayers, which dominates at large $q$, together with a bending term at small $q$, and a weak protrusion term in the intermediate $q$ regime, which was later considered negligible. In general, it is clear that different membrane models, each with its own set of internal parameters, may be used to fit the simulation results for the elasticity of the membrane. The main difficulty with all these approaches is the separation of the coupled (when the membrane layers fluctuate as a single unit) and the uncoupled (when they fluctuate independently) regimes.

In this paper, we propose a general approach to analyze computer simulation data. Our approach requires the construction of a surface, which interpolates the head groups in each membrane layer. The method provides a route to isolate the coupled modes, hence enabling the computation of elastic constants using small membrane areas. This is a desirable feature as it expands the capabilities of computer simulations to investigate more accurate atomistic models. Coarsegrained models allow the computation of very large systems and long time scales, but some important microscopic aspects of the membranes can only be understood with more detailed atomistic models that incorporate detailed information on the lipid chain length and saturation. Moreover, these models take into account screening effects mediated by water and ions more accurately, and predict phospholipid diffusion coefficient of the right order of magnitude, unlike mesoscopic models which tend to overestimate them by several orders of magnitude. ${ }^{33}$ Because the number of sites in atomistic model is much larger, and shorter times steps are needed to generate stable trajectories, most atomistic simulations are restricted to relatively small membrane areas $\left(\sim 10^{2} \mathrm{~nm}^{2}\right)$. Therefore, the accessible wavevectors are only slightly below the $2 \pi / d$ $\sim 1 \mathrm{~nm}^{-1}$ threshold, leaving a very narrow range of useful $q$ vectors that can be used to quantify the mesoscopic fluctuation regime. This fact makes it difficult to accurately compute the bending modulus from simulations of systems with small cross-sectional areas.

The aim of this work is to find a better description of the phospholipid membrane undulatory mode. The main challenge here is to disentangle the mixing of the high-q protrusions and the mesoscopic undulations, without imposing a prefixed wavevector threshold that separates them. To achieve this goal, we introduce a new coupled undulatory (CU) mode which describes the correlated movement of the two monolayers. The amplitude of this mode decays to zero when the fluctuations of the two monolayers are uncoupled. By combining this mode with the usual peristaltic one, we get a full description of the fluctuations of symmetric bilayers over the whole range of $q$ vectors. The main features of this new description are:

- The physical description of the new coupled undulatory mode is much simpler than the usual undulatory mode that is gradually merged into the uncoupled protrusions. ${ }^{6,23}$

- The fit of the simulation results provides a more robust estimation of the bending rigidity, even from the relatively large wavevectors sampled in simulations with small membrane areas. 
TABLE I. Simulation parameters of the systems investigated in this work. $\gamma_{0}$ is the surface tension, $L_{\alpha}$ the box length in direction $\alpha$, $A_{\text {Phos }}$ is the area per phospholipid, $\mathrm{N}_{\mathrm{Phos}}$ the number of phospholipid molecules per layer, $\mathrm{N}_{\text {Water }}$ number of water molecules, $\Delta T$ production simulation time, and $\mathrm{N}_{\mathrm{CW}}$ the number of configurations employed in the fluctuation analysis. $\left(\mathrm{N}, \gamma_{0}\right)$ is the ensemble to fix surface tension and $\left(\mathrm{N}, A_{0}, \mathrm{~T}\right)$ to fix projected area. The asterisk in $\left(\mathrm{N}, \gamma_{0}\right)^{*}$ indicates the simulation performed with the weak coupling barostat. $\left\langle L_{z}\right\rangle \approx 14.0 \mathrm{~nm}$ for all systems.

\begin{tabular}{|c|c|c|c|c|c|c|c|c|}
\hline Ensemble & $\begin{array}{c}\gamma_{0} \\
(\mathrm{mN} / \mathrm{m})\end{array}$ & $\begin{array}{l}\left\langle L_{x}\right\rangle \\
(\mathrm{nm})\end{array}$ & $\begin{array}{c}\left\langle L_{y}\right\rangle \\
(\mathrm{nm})\end{array}$ & $\begin{array}{l}A_{\text {Phos }} \\
\left(\mathrm{nm}^{2}\right)\end{array}$ & $\mathrm{N}_{\text {Phos }}$ & $\mathrm{N}_{\text {Water }}$ & $\begin{array}{l}\Delta T \\
\mu \mathrm{s}\end{array}$ & $\mathrm{N}_{\mathrm{CW}}$ \\
\hline \multicolumn{9}{|c|}{ POPC } \\
\hline$\left(\mathrm{N}, \gamma_{0}, \mathrm{~T}\right)$ & 0 & $12.73 \pm 0.01$ & $13.39 \pm 0.02$ & $0.666 \pm 0.02$ & 256 & 13250 & 7.5 & 9980 \\
\hline$\left(\mathrm{N}, \gamma_{0}, \mathrm{~T}\right)$ & 0 & $25.16 \pm 0.01$ & $26.46 \pm 0.02$ & $0.666 \pm 0.001$ & 1000 & 57000 & 3.975 & 5301 \\
\hline$\left(\mathrm{N}, \gamma_{0}, \mathrm{~T}\right)$ & 0 & $50.27 \pm 0.01$ & $52.88 \pm 0.02$ & $0.665 \pm 0.001$ & 4000 & 228000 & 2.1 & 10000 \\
\hline$\left(\mathrm{N}, \gamma_{0}, \mathrm{~T}\right)$ & $15.4 \pm 0.9$ & $25.85 \pm 0.01$ & $27.19 \pm 0.01$ & $0.703 \pm 0.001$ & 1000 & 57000 & 7.5 & 20000 \\
\hline$\left(\mathrm{N}, \gamma_{0}, \mathrm{~T}\right)$ & $27.3 \pm 1.1$ & $26.58 \pm 0.01$ & $27.86 \pm 0.01$ & $0.744 \pm 0.001$ & 1000 & 57000 & 7.5 & 20000 \\
\hline$\left(\mathrm{N}, \gamma_{0}, T\right)^{*}$ & 0 & $50.23 \pm 0.01$ & $52.93 \pm 0.02$ & $0.665 \pm 0.001$ & 4000 & 228000 & 2.1 & 10000 \\
\hline$\left(\mathrm{N}, A_{0}, \mathrm{~T}\right)$ & & 50.27 & 52.88 & 0.666 & 4000 & 228000 & 2.1 & 10000 \\
\hline \multicolumn{9}{|c|}{ DPPC } \\
\hline$\left(\mathrm{N}, \gamma_{0}, \mathrm{~T}\right)$ & 0 & $25.24 \pm 0.01$ & $25.81 \pm 0.02$ & $0.636 \pm 0.001$ & 1024 & 53000 & 7.5 & 10000 \\
\hline
\end{tabular}

- Moreover, our method provides a route to discuss a very important open question, namely, what is the connection between the surface tension obtained from the coefficient $q^{2}$ in the fluctuation spectrum of the coupled undulatory and the surface tension imposed on the flat membrane via a suitable barostat.

- We have also refined the analysis of the peristaltic mode, by separating the bulk and surface contributions to this mode, and by taking into account the smooth transition of the bulk contribution from a solid-like elasticity to a liquid-like plasticity, which occurs as the wavevector $q$ increases.

To illustrate and test the methodology, we have performed simulations of bilayer membranes using coarsegrained models.

\section{MODEL AND SIMULATION DETAILS}

As model system we have chosen a POPC (1-palmitoyl2-oleoyl-sn-glycero-3-phosphocholine) bilayer. Phosphatidylcholines are major components in many biological membranes, often playing a major role in supporting transmembranes proteins. We use a coarse-grained model, where the phospholipid is modeled as a collection of beads joined by rigid bonds, angular and dihedral terms. In recent years, many mesoscopic models have been introduced. ${ }^{34-37}$ All of them share some commonalities in the approach followed to coarse grain the phospholipid. In this work, we use the MARTINI force-field, which has been successfully parametrized to model a wide range of phospholipids. ${ }^{38}$ Our simulations were performed at $320 \mathrm{~K}$, hence, it was not necessary to use the antifreeze parameters to avoid water freezing, which for MARTINI occurs between 280 and $300 \mathrm{~K}$. POPC features a lower melting temperature, so that we investigate the fluctuations of the membrane in the liquid crystal phase. We truncated and shifted the Lennard-Jones non-bonding short range interactions at $0.9 \mathrm{~nm}$. A shifted coulomb potential with a cutoff of $1.2 \mathrm{~nm}$ and an effective dielectric constant of 15 were employed to model the electrostatic interactions associated to the charges in the POPC head group.
The basic systems consisted of $N_{\text {Phos }}=1000$ phospholipid molecules per layer and $N_{\text {Water }}=57000$ coarse grained water molecules. Periodic boundary conditions were applied in all directions and the motion of center of mass was removed every 10 time steps. We employed two Berendsen thermostats with a coupling constant of 2 ps to maintain the temperature of the bilayer and the solvent at the desired temperature. We also used a Berendsen semi-isotropic barostat to simulate systems at different surface tensions. The plane of the bilayer $(x, y)$ was coupled to a barostat with a coupling constant of 3 ps and compressibility $4 \times 10^{-5} \mathrm{bar}^{-1}$, while the box axis normal to the bilayer plane, $z$, was coupled to another barostat with the same time constant and compressibility $10^{-4}$ bar $^{-1}$ and 1 bar pressure. We investigated membranes with surface tensions in the range $0-27.3 \mathrm{mN} / \mathrm{m}$. For these surface tensions, we did not observe the formation of large holes in the membrane. The simulation time step was set to 0.03 ps. A typical simulation involved $0.5 \mu$ s equilibration and several $\mu$ s (see $\Delta T$ in Table I) production. We saved $\mathrm{N}_{\mathrm{CW}}$ configurations for the fluctuation analysis. To analyze the dependence of the results with the system size, we performed simulations for different membrane cross-sectional areas. The water to phospholipid ratio was kept fixed in these simulations and equal to the ratio employed in the $\mathrm{N}_{\mathrm{Phos}}$ $=1000$ simulations. To analyze the dependence of our results with the surface tension coupling scheme $\gamma_{0}$, we performed additional simulations with a weak coupling barostat set at 50 ps vs 3 ps, employed in the other simulations. Further, we performed a simulation at constant area, $\left(\mathrm{N}, A_{0}, \mathrm{~T}\right)$, which was set equal to the mean value $\left\langle A_{0}\right\rangle$ obtained from the tensionless simulation. Finally, using the MARTINI force-field, we simulated the DPPC bilayer membrane in order to compare our results with those reported by Watson et al. ${ }^{31}$ Details on the simulations parameters are given in Table I. All the simulations were performed with the GROMACS 4.5 simulation package. ${ }^{39}$

The surface tensions were computed through the microscopic pressure tensor route,

$$
\gamma_{0}=L_{z}\left[P_{z z}-\frac{1}{2}\left(P_{x x}+P_{y y}\right)\right]
$$


where $P_{\alpha \beta}$ are the pressure tensor components with $\alpha$ and $\beta$ being the cartesian coordinates, $(x, y, z)$, and $L_{z}$ the box length in the direction normal to the bilayer plane. The pressure components were obtained from the virial equation,

$$
P_{\alpha \beta} V=\sum_{i=1}^{N} m_{i} \mathbf{v}_{i, \alpha} \mathbf{v}_{i, \beta}+\sum_{i=1}^{N-1} \sum_{j>i}^{N} \mathbf{r}_{i j, \alpha} \mathbf{f}_{i j, \beta},
$$

where $\mathbf{v}_{i, \alpha}$ represents the velocity of atom $i$ in direction $\alpha, \mathbf{r}_{i j}$ is the vector joining atoms $i$ and $j$, and $\mathbf{f}_{i j}$ is the force between these two atoms.

In the rest of the paper, we will use the average distance between the phosphate groups in the tensionless membrane, $\sigma=\sqrt{A_{\text {Phos }}}=0.816 \mathrm{~nm}$ for the POPC and 0.7977 for the DPPC, as the unit length, and $k T=\beta^{-1}$ as the unit of energy, in order to present our results in reduced units.

\section{FLUCTUATION MODES OF BILAYER MEMBRANES}

\section{A. Instantaneous shape $\xi(R)$ of the membrane}

The analysis of the thermal fluctuations is a very powerful approach to study the elastic properties of membranes. All the relevant modes from the mesoscopic to the molecular scale, can be sampled using a single computer simulation. Different methods have been used to describe the fluctuation spectrum of a membrane, ${ }^{40}$ and they all should agree in the macroscopic elastic limit described by the Helfrich Hamiltonian. ${ }^{4}$ However, in the range between mesoscopic and molecular scales we expect deviations from that simple behavior, since the fluctuations include changes in the internal structure of the bilayer, and not only in the undulations of the mathematical surface, $z=\xi(x, y) \equiv \xi(\mathbf{R})$, used to describe the instantaneous shape (IS) of the membrane. To explore the fluctuation spectrum in a computer simulation, we have to establish a connection between the molecular structure of the membrane (i.e., the bilayer of phospholipid molecules) and its mathematical IS. The problem is somehow similar to the study of the capillary fluctuations at liquid-vapor surfaces, where $z=\xi(\mathbf{R})$ is the intrinsic surface used in the capillary wave theory. ${ }^{41-43}$ Over the last decade, there have been important advances in the characterization of $\xi(\mathbf{R})$ and its fluctuations, which can be done through the analysis of independent molecular configurations. ${ }^{44-52}$ In this paper, we take advantage of these advances to quantify the fluctuation modes of biological membranes.

The construction of the IS starts with the selection of a set of pivots at specific atomic positions. For liquid-vapor surfaces, the number of pivots per unit area has to be carefully chosen, ${ }^{53}$ but in the case of a bilayer membrane this problem is simpler, as the phospholipids do not leave their corresponding monolayer during the duration of the simulation. Hence, we choose the phosphate groups as the pivots of the hereafter referred as upper and lower monolayers in the bilayer membrane. ${ }^{54}$ The mathematical surfaces $z=\xi^{\text {up, low }}(\mathbf{R})$ that represent the instantaneous shape of each of these monolayers are defined by constructing an interpolating function that passes through the positions of the pivots. Notice that once the atomic pivots have been chosen, there is some arbitrariness in defining the interpolation scheme used to construct $\xi^{\text {up, low }}(\mathbf{R})$ meaning that for a given set of pivots several IS surfaces can be employed. In this work, we have employed an interpolation scheme based on a two-dimensional Delaunay triangulation (DT). The DT is performed using the pivots' (i.e., the phosphate groups in this work) coordinates in the membrane plane $(\mathrm{x}, \mathrm{y})$. With the DT we identify the nearest neighbours of each phosphate group. This information is then used to construct a three-dimensional triangulated surface, where the triangle edges join each pivot to its nearest neighbours. The procedure has been discussed in Ref. 53 for the liquid-vapor interface and in Refs. 54-56 for the specific case of surfactant monolayers and foam surfactant bilayers. Other approaches can be used to construct the intrinsic surface as we discuss in the supplementary material. ${ }^{58}$

Considering the usual periodic boundary conditions of the simulation cell, with lengths $L_{x}$ and $L_{y}$ on the bilayer plane, we represent the IS in terms of its Fourier components

$$
\xi\left(\mathbf{R}, q_{u}\right)=\sum_{|\mathbf{q}| \leq q_{u}} \hat{\xi}_{q} e^{i \mathbf{q} \mathbf{R}} .
$$

The wavevectors defined are as $\mathbf{q}=2 \pi\left(n_{x} / L_{x}, n_{y} / L_{y}\right)$, for $n_{x, y}=0, \pm 1, \pm 2, \ldots$ Because we perform simulations at constant surface tension, the box cross-sectional area and by extension the lattice of reciprocal vectors q fluctuate. However, for the box areas sampled in the equilibrium state, the changes in $\mathbf{q}$ are small. Hence, we accumulate all the relevant statistics for each Fourier term $\left(n_{x}, n_{y}\right)$ under the same $\mathbf{q}$ vector, so that the mean square modulus is given by $\left\langle q^{2}\right\rangle \approx$ $(2 \pi)^{2}\left(n_{x}^{2}+n_{y}^{2}\right) / A_{0}$, where $A_{0}=\left\langle L_{x} L_{y}\right\rangle$ is the mean crosssectional area. The upper limit, $q_{u}=2 \pi / \lambda_{c}$ in (3) is defined by the wavelength cutoff, $\lambda_{c} \approx \sqrt{A_{0} / N_{\text {Phos }}}=\sqrt{A_{\text {Phos }}}$, i.e., by the average distance between the lipids phosphate groups, where $N_{s}$ is the number of lipid molecules per monolayer. Notice that to describe the IS corrugations beyond this minimum distance would not be physically meaningful. The instantaneous shape of each monolayer provides information on both the undulations of the membrane as a whole and on the fluctuations of the membrane thickness. In a symmetric bilayer, the fluctuations of $\xi^{\text {up }}(\mathbf{R})$ and $\xi^{\text {low }}(\mathbf{R})$ should be statistically similar. It is then possible to average out the independent results for the two monolayers, $(m)$, to get better statistics. The sampling of the IS for a large number of configurations along an equilibrated computer simulation run, gives for each configuration the amplitudes $\hat{\xi}_{q}^{\text {up }}$ and $\hat{\xi}_{q}^{\text {low }}$ of the fluctuating modes. ${ }^{44}$

\section{B. Fluctuation modes}

The usual description of the fluctuations in bilayer membranes ${ }^{57}$ is done in terms of the eigenvalues of the matrix

$$
\frac{1}{2}\left(\begin{array}{cc}
\left\langle\left|\hat{\xi}_{q}^{\text {low }}\right|^{2}\right\rangle & \left\langle\hat{\xi}_{q}^{\text {low }} \hat{\xi}_{q}^{\text {up } *}\right\rangle \\
\left\langle\hat{\xi}_{q}^{\text {low } \left.* \hat{\xi}_{q}^{\text {up }}\right\rangle}\right. & \left\langle\left|\hat{\xi}_{q}^{\text {up }}\right|^{2}\right\rangle
\end{array}\right),
$$

which is constructed using the Fourier components of the surfaces associated to each monolayer, upper and lower. The softer undulatory (U) mode, ${ }^{5,57}$ corresponds to the highest eigenvalue. It describes the fluctuations of the mean surface, 


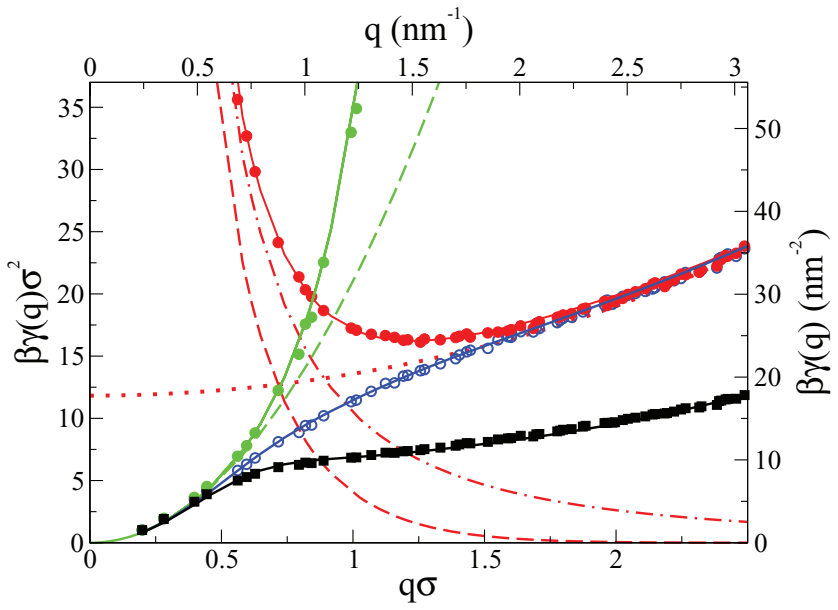

FIG. 1. The surface tension as function of the wavevector $q$ for the POPC tensionless membrane with $N_{\text {Phos }}=1000$. The symbols represent our simulation results: grey (green) full circles the coupled undulatory mode $\gamma^{\mathrm{CU}}(q)$; dark (red) full circles the peristaltic-protrusion mode $\gamma_{\text {eff }}^{\mathrm{P}}(q)$; empty (blue) circles the undulatory mode $\gamma^{\mathrm{U}}(q)$; and black squares the mean value obtained from the lower and upper monolayers $\gamma^{\mathrm{m}}(q)$. The solid lines joining the symbols represent the fitting to the simulation data: Eq. (11) for the coupled undulatory mode, Eq. (13) for the peristaltic-protrusion mode; Eq. (16) for the undulatory mode; and Eq. (18) for the monolayer fluctuations. The grey (green) dashed line represents the parabolic low $q$ behavior of the coupled mode $\gamma^{\mathrm{CU}}(q)$. The dark (red) dashed-dotted line is the elastic contribution to the peristaltic mode $\gamma_{e f f}^{\mathrm{P}}(q)$ without tails fluidization $u_{0}^{\mathrm{P}} / q^{2}$. The result corresponding to tail fluidization $u_{0}^{\mathrm{P}} / q^{2} \exp \left(-\left(q / q_{e l}^{\mathrm{P}}\right)^{2}\right)$ is given by the dark (red) dashed curve. The dotted (red) line is the fit to the protrusion surface tension contribution $\gamma^{\mathrm{P}}(q)$.

which lies between the two identical lipid monolayers,

$$
\begin{aligned}
\left\langle\left|\hat{\xi}_{q}^{\mathrm{U}}\right|^{2}\right\rangle & =\left\langle\left|\frac{\hat{\xi}_{q}^{\text {low }}+\hat{\xi}_{q}^{\mathrm{up}}}{2}\right|^{2}\right\rangle \\
& =\frac{1}{2}\left\langle\left|\hat{\xi}_{q}^{\mathrm{m}}\right|^{2}\right\rangle+\frac{1}{2}\left\langle\hat{\xi}_{q}^{\mathrm{low}} \hat{\xi}_{q}^{\mathrm{up} *}\right\rangle,
\end{aligned}
$$

where we use $\left\langle\left|\hat{\xi}_{q}^{\mathrm{m}}\right|^{2}\right\rangle=\left\langle\left|\hat{\xi}_{q}^{\text {up }}\right|^{2}\right\rangle=\left\langle\left|\hat{\xi}_{q}^{\text {low }}\right|^{2}\right\rangle$.

The lowest eigenvalue of (4) corresponds to the peristaltic $^{23}(\mathrm{P})$ mode and it describes the fluctuations of the membrane (half) thickness

$$
\begin{aligned}
\left\langle\left|\hat{\xi}_{q}^{\mathrm{P}}\right|^{2}\right\rangle & =\left\langle\left|\frac{\hat{\xi}_{q}^{\text {low }}-\hat{\xi}_{q}^{\mathrm{up}}}{2}\right|^{2}\right\rangle \\
& =\frac{1}{2}\left\langle\left|\hat{\xi}_{q}^{\mathrm{m}}\right|^{2}\right\rangle-\frac{1}{2}\left\langle\hat{\xi}_{q}^{\mathrm{low}} \hat{\xi}_{q}^{\mathrm{up} *}\right\rangle .
\end{aligned}
$$

In our view, the main problem with the description given above is that (as shown in Fig. 1) the U (or mean surface) mode exhibits a complex behavior, from the low q coupled regime with $\left\langle\hat{\xi}_{q}^{\text {low }} \hat{\xi}_{q}^{\text {up* }}\right\rangle \approx\left\langle\left|\hat{\xi}_{q}^{\mathrm{m}}\right|^{2}\right\rangle$, to the uncoupled regime, at high $q$ with $\left\langle\hat{\xi}_{q}^{\text {low }} \hat{\xi}_{q}^{\text {up* }}\right\rangle \approx 0$ and $\left\langle\left|\hat{\xi}_{q}^{\mathrm{U}}\right|^{2}\right\rangle \approx\left\langle\left|\hat{\xi}_{q}^{\mathrm{P}}\right|^{2}\right\rangle$, which is usually referred to as monolayer protrusions. ${ }^{24}$ The existence of these two regimes prevents a simple physical description of $\left\langle\left|\hat{\xi}_{q}^{\mathrm{U}}\right|^{2}\right\rangle$ over the whole range of $q$. Therefore, Eq. (5) is usually described as a piece-wise function of $q$, with different fitting forms for low and high wavevectors.
So, the main idea of the present work is to describe the whole fluctuation matrix (4) with the usual definition of the peristaltic mode, Eq. (6), and substitute the undulatory mode, Eq. (5), by the CU mode described by

$$
\left\langle\left|\hat{\xi}_{q}^{\mathrm{CU}}\right|^{2}\right\rangle=\left\langle\hat{\xi}_{q}^{\text {low }} \hat{\xi}_{q}^{u p *}\right\rangle=\left\langle\left|\hat{\xi}_{q}^{\mathrm{U}}\right|^{2}\right\rangle-\left\langle\left|\hat{\xi}_{q}^{\mathrm{P}}\right|^{2}\right\rangle .
$$

The CU mode is zero when the fluctuations of the two layers are uncorrelated (high $q$ ), and equal to the amplitude of the fluctuations of the monolayers $\left\langle\left|\hat{\xi}_{q}^{\mathrm{CU}}\right|^{2}\right\rangle=\left\langle\hat{\xi}_{q}^{\text {low }} \hat{\xi}_{q}^{\text {up } *}\right\rangle$ $\approx\left\langle\left|\hat{\xi}_{q}^{\mathrm{m}}\right|^{2}\right\rangle \approx\left\langle\left|\hat{\xi}_{q}^{\mathrm{U}}\right|^{2}\right\rangle$, when the fluctuations of both monolayers are fully correlated (low $q$ ). This mode does not include protrusions which, as we will see, will allow us to make a simple physical description of the membranes fluctuations over the whole range of $q$.

Another important difference with previous studies of membranes fluctuations is that they focus on the mean square amplitude of the fluctuations $\left\langle\left|\hat{\xi}_{q}^{\mathrm{X}}\right|^{2}\right\rangle$. However, in this section we have preferred, as is usual in the study of the capillary waves of the liquid surfaces, to focus on the elastic energy of each mode which is proportional to the inverse of $\left\langle\left|\hat{\xi}_{q}^{\mathrm{X}}\right|^{2}\right\rangle$. Although both approaches provide similar information in the investigation of tensionless membranes, the method used here is superior when investigating membranes under tension. Indeed this approach enables a detailed study and modelization of the different energetic contributions to the $(\mathrm{CU})$ and $(\mathrm{P})$ modes, and provides a simple mechanic analogy between the fluctuations of the membrane and the fluctuations of an arrangement of springs. However, in Sec. IV B we will present our results using $\left\langle\left|\hat{\xi}_{q}^{\mathrm{X}}\right|^{2}\right\rangle$ in order to connect better with previous works.

Following the approach used in the investigation of capillary waves at liquid surfaces, we assume that the equipartition for the mean elastic energy of each fluctuation mode is fulfilled and represented by $q^{2} \gamma^{\mathrm{x}}(q) A_{0}\left\langle\left|\hat{\xi}_{q}^{\mathrm{x}}\right|^{2}\right\rangle / 2=k T / 2$, where $\gamma^{\mathrm{x}}(q)$ is a q-dependent surface tension,

$$
\gamma^{\mathrm{x}}(q)=\frac{k_{\mathrm{B}} T}{q^{2}\left(\left|\hat{\xi}_{q}^{\mathrm{x}}\right|^{2}\right\rangle A_{0}},
$$

where " $\mathrm{x}$ " indicates that the method may be applied to any choice of the surface $\xi^{\mathrm{x}}(\mathbf{R}),(\mathrm{m}, \mathrm{P}, \mathrm{U}$, or $\mathrm{CU}$, taken as representative of the instantaneous shape of the membrane. In Fig. 1, we present the simulation results for the two most obvious choices, $\gamma^{\mathrm{m}}(q)$ associated to the phosphate groups in one of the monolayers, and $\gamma^{\mathrm{U}}(q)$ obtained from the usual definition of the undulations, i.e., from the mean position of the bilayer. Fig. 1 shows that $\gamma^{\mathrm{m}}(q)$ and $\gamma^{\mathrm{U}}(q)$ have similar values for $q \sigma \leq 0.5$. This $q$ dependence of the surface tension is consistent with the parabolic shape

$$
\gamma^{\mathrm{x}}(q)=\gamma_{0}+\kappa q^{2}+\mathcal{O}^{\mathrm{x}}\left(q^{4}\right)
$$

as predicted by the surface hamiltonian, ${ }^{4,5}$ in terms of the macroscopic surface tension $\left(\gamma_{0}=0\right.$ for the tensionless state presented in Fig. 1) and the bending modulus $\kappa$.

However, for larger wavevectors, $\gamma^{\mathrm{m}}(q)$ and $\gamma^{\mathrm{U}}(q)$ differ due to the contributions from the internal degrees of freedom of the bilayer membrane. The swaying slopes of $\gamma^{\mathrm{x}}(q)$ signal the crossover from the undulating regime, in which the membrane fluctuates as a whole, to the independent 


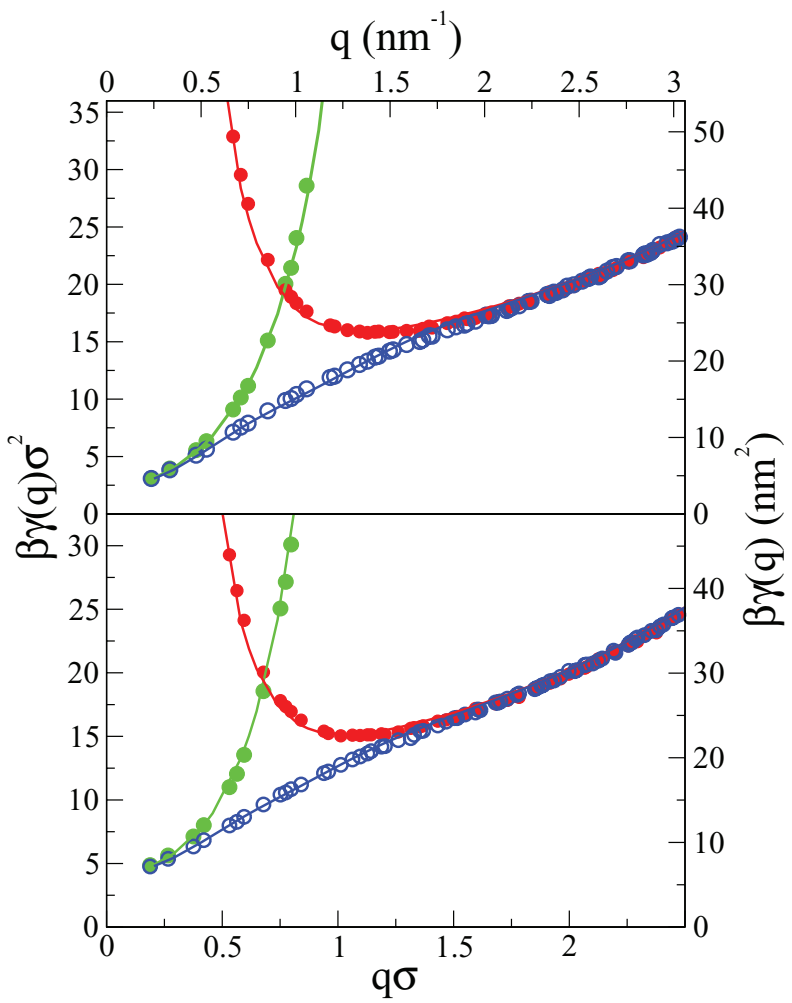

FIG. 2. The surface tensions as function of the wavevector $q$ for POPC membranes under tension with $N_{\text {Phos }}=1000$. (Top panel) $\gamma_{0}=15.3 \mathrm{mN} / \mathrm{m}$, (bottom) $\gamma_{0}=27.3 \mathrm{mN} / \mathrm{m}$. The symbols show our simulation results: Grey (green) full circles the coupled undulatory mode $\gamma^{\mathrm{CU}}(q)$; dark (red) full circles the peristaltic-protrusion mode $\gamma_{\text {eff }}^{\mathrm{P}}(q)$; and empty (blue) circles the undulatory mode $\gamma^{\mathrm{U}}(q)$. The solid lines represent the fittings to the simulation data to Eq. (11) for the coupled undulatory mode, Eq. (13) for the peristalticprotrusion mode, and Eq. (16) for the undulatory mode.

fluctuations of each monolayer at high $q$. In the following, we discuss our simple description of the fluctuations, where the elastic energy is separated into two elementary modes: the coupled undulatory and the peristaltic modes. Note that the coupled undulatory mode is free of protrusions while the peristaltic includes low-q peristaltic like fluctuations and high-q protrusions.

\section{The coupled undulatory mode}

For the CU mode described by $\left\langle\hat{\xi}_{q}^{l o w} \hat{\xi}_{q}^{u p *}\right\rangle$, the wavevector dependent surface tension is given by

$$
\gamma^{\mathrm{CU}}(q)=\frac{k_{\mathrm{B}} T}{q^{2}\left(\hat{\xi}_{q}^{\text {low }} \hat{\xi}_{q}^{\text {pp* }}\right\rangle A_{0}} .
$$

As shown in Fig. 2, the simulation results for $\gamma^{\mathrm{CU}}(q)$ are identical to those of $\gamma^{\mathrm{U}}(q)$ and $\gamma^{\mathrm{m}}(q)$ in the low $q$ regime, $q \sigma \lesssim 0.5$. They all follow the expansion (9), for the undulations of the membrane as a whole. However, increasing the wavevector above $q \sigma \gtrsim 0.5$ the value of $\left\langle\hat{\xi}_{q}^{\text {low }} \hat{\xi}_{q}^{u p *}\right\rangle$ decays very rapidly, so that $\gamma^{\mathrm{CU}}(q)$ grows faster than $\kappa q^{2}$. This is in contrast with the softer behavior of $\gamma^{\mathrm{U}}(q)$ and $\gamma^{\mathrm{m}}(q)$, which includes negative contributions from the $q^{4}$ and higher order terms, appearing in the expansion (9). As shown in Fig. 2, the problem is even more evident for POPC membranes kept under tension. For $\gamma_{0}=25.51 \mathrm{mN} / \mathrm{m}$, the results for $\gamma^{\mathrm{U}}(q)$ within our simulation box would apparently be better fitted by a straight line, instead of the parabolic function given in Eq. (9). The negative contributions of the $q^{4}$ and higher order terms are already significant for $q \sigma \lesssim 0.5$, making difficult the estimation of $\kappa$. The analysis of the CU mode, Eq. (10) provides a much more accurate estimation of the two coefficients, surface tension and bending modulus. Fitting the low q limit of $\gamma^{\mathrm{CU}}(q)$ to Eq. (9), we get surface tensions, $\gamma_{0}$, in excellent agreement with the surface tensions imposed in the simulations. The bending modulus takes similar values for the three surface tensions, $\gamma_{0}$, investigated in this work (see Table II).

We may go one step further in the characterization of $\gamma^{\mathrm{CU}}(q)$, by noting that the full range of $q$ values of this function, from $\gamma^{\mathrm{CU}}(0)=\gamma_{0}$ to $\gamma^{\mathrm{CU}}(q) \gtrsim 130 k T / \sigma^{2}$, can be very well fitted by the empirical formula,

$$
\gamma^{\mathrm{CU}}(q)=\gamma_{0}+\kappa q^{2}\left(1+\left(\frac{q}{q_{\mathrm{u}}^{\mathrm{CU}}}\right)^{\alpha^{\mathrm{CU}}}\right),
$$

where the term in brackets represents the molecular size effects that signal the limits of Helfrich mesoscopic Hamiltonian. For all membranes analyzed $\alpha^{\mathrm{CU}} \sigma>4.0$ which means a continuous (but very rapid) increase of $\gamma^{\mathrm{CU}}(q)$ for values

TABLE II. Fitted parameters for the coupled undulatory $\gamma^{\mathrm{CU}}(q)$ and peristaltic-protrusion $\gamma_{e f f}^{\mathrm{P}}(q)$ modes. The parameter $\gamma_{0}$ is fixed to the simulated macro-

\begin{tabular}{|c|c|c|c|c|c|c|c|c|c|c|}
\hline \multirow[b]{2}{*}{$\gamma_{0}(\mathrm{mN} / \mathrm{m})$} & \multirow[b]{2}{*}{$d(\mathrm{~nm})$} & \multirow[b]{2}{*}{$d / \sigma$} & \multicolumn{4}{|c|}{ Coupled undulatory mode } & \multicolumn{4}{|c|}{ Peristaltic-protrusion mode } \\
\hline & & & $\beta \gamma_{0} \sigma^{2}$ & $\beta \kappa$ & $q_{u}^{\mathrm{CU}} \sigma$ & $\alpha^{\mathrm{CU}}$ & $\beta u_{0}^{\mathrm{P}}\left(\mathrm{nm}^{-4}\right)$ & $q_{e l}^{\mathrm{P}} \sigma$ & $\beta \gamma_{0}^{\mathrm{P}} \sigma^{2}$ & $\beta \kappa^{\mathrm{P}}$ \\
\hline \multicolumn{11}{|c|}{ POPC } \\
\hline 0 & 4.24 & 5.19 & 0.0 & $20.9 \pm 1$ & $\begin{array}{l}1.10 \pm 0.15 \\
(1.21)\end{array}$ & $4.57 \pm 0.2$ & $26.0 \pm 1.0$ & $0.83 \pm 0.05$ & $12.6 \pm 1.2$ & $1.81 \pm 0.2$ \\
\hline 15.2 & 4.09 & 5.01 & 2.26 & $21.7 \pm 1.5$ & $\begin{array}{l}0.97 \pm 0.15 \\
(1.26)\end{array}$ & $4.77 \pm 0.2$ & $23.8 \pm 1.0$ & $0.67 \pm 0.05$ & $14.0 \pm 1.2$ & $1.63 \pm 0.2$ \\
\hline 27.51 & 3.93 & 4.82 & 4.12 & $20.9 \pm 2.5$ & $\begin{array}{l}0.98 \pm 0.15 \\
(1.29)\end{array}$ & $4.28 \pm 0.2$ & $20.1 \pm 1.0$ & $0.63 \pm 0.05$ & $12.9 \pm 1.2$ & $1.81 \pm 0.2$ \\
\hline \multicolumn{11}{|c|}{ DPPC } \\
\hline 0 & 4.06 & 5.09 & 0.0 & $23.0 \pm 1$ & $\begin{array}{l}1.09 \pm 0.15 \\
(1.23)\end{array}$ & $4.82 \pm 0.2$ & $26.7 \pm 1.0$ & $0.69 \pm 0.05$ & $13.3 \pm 1.2$ & $1.26 \pm 0.2$ \\
\hline
\end{tabular}
scopic surface tension. In the column corresponding to $q_{u}^{\mathrm{CU}} \sigma$, we show (in brackets) the values of $2 \pi / d$, where $d$ is the mean distance between layers. 
of $q$ above the cutoff $q_{\mathrm{u}}^{\mathrm{CU}}$. This high value of $\alpha^{\mathrm{CU}}$ prevents a contamination of our fit of the parabolic dependence in (9) at lower values of $q$. Table II lists the best fits for the cutoff, which are lower than $q_{\mathrm{u}}^{\mathrm{CU}}=2 \pi / d$, where $d=\left\langle\hat{\xi}_{0}^{\text {up }}-\hat{\xi}_{0}^{\text {low }}\right\rangle$ is the mean distance between layers. This is in agreement with the physical intuition, since the undulations of the membrane as a whole should be restricted to wavelengths longer than the thickness of the membrane. $q_{\mathrm{u}}^{\mathrm{CU}}=2 \pi / d$ is otherwise the cutoff that has been traditionally adopted to define the threshold that separates the undulation and the protrusions behavior. ${ }^{6,23}$ With our choice of the CU mode the separation of the two regimes is already built in the function $\gamma^{\mathrm{CU}}(q)$. This function becomes very large for $q>q_{\mathrm{u}}^{\mathrm{CU}}$, showing that $\left\langle\hat{\xi}_{q}^{\text {low }} \hat{\xi}_{q}^{u p *}\right\rangle$ features a fast and continuous decay for larger $q$ vectors.

\section{Physical description of the peristaltic mode}

The energy of an undulating mode, with wavevector $q$ and amplitude $\hat{\xi}_{q}^{\mathrm{x}}$, is described by $U^{\mathrm{x}}(q)$ $=q^{2} \gamma^{\mathrm{x}}(q)\left|\hat{\xi}_{q}^{\mathrm{x}}\right|^{2} A_{0} / 2$ in terms of the surface tension defined in Eq. (9), showing that the low $q$ modes are very soft, with $u^{\mathrm{x}}(q) \sim \gamma_{0} q^{2}$ for membranes under tension, and even softer, $u^{\mathrm{x}}(q) \sim \kappa q^{4}$ for tensionless membranes. It is well known ${ }^{6,23}$ that the peristaltic mode (Eq. (6)) does not become increasingly softer in the $q=0$ limit. There is an elastic energy that opposes the expansion or compression of the volume contained between the two monolayers, which is filled by the aliphatic tails of the phospholipid molecules. The bulk elastic modulus may be extracted from the mean square fluctuations of the $q=0$ Fourier component of the $\mathrm{P}$ mode, $\left\langle\left|\hat{\xi}_{0}^{\mathrm{P}}\right|^{2}\right\rangle$, sampled in our computer simulations. The energy of this mode is described in terms of $u_{0}^{\mathrm{P}} \equiv k_{\mathrm{B}} T /\left(A_{0}\left\langle\left|\hat{\xi}_{0}^{\mathrm{P}}\right|^{2}\right\rangle\right)$. In addition to the bulk contribution, the fluctuations of $\hat{\xi}_{q}^{\mathrm{P}}$ describe also the independent corrugations of the upper and lower surfaces, or protrusions, reflected in a surface contribution, which is proportional to $q^{2}$, and possibly higher order terms.

Adding the bulk and surface contributions, as two springs set in parallel, the energy of a $\mathrm{P}$ mode fluctuation with amplitude $\hat{\xi}_{q}$ would be given by $U^{\mathrm{P}}(q)=\left[u^{\mathrm{P}}(q)\right.$ $\left.+q^{2} \gamma^{\mathrm{P}}(q)\right]\left|\hat{\xi}_{q}^{\mathrm{P}}\right|^{2} A_{0} / 2$, where $u^{\mathrm{P}}(q)=u_{0}^{\mathrm{P}}+\mathcal{O}\left(q^{2}\right) \quad$ and $\gamma^{\mathrm{P}}(q)=\gamma_{0}^{\mathrm{P}}+\kappa^{\mathrm{P}} q^{2}+\mathcal{O}\left(q^{4}\right)$, describe each contribution as functions of $q$. For low $q$, the relevant contribution comes from the bulk, and from the equipartition of the energy we expect $\left\langle\left|\hat{\xi}_{q}^{\mathrm{P}}\right|^{2}\right\rangle A_{0} \approx k T / u_{0}^{\mathrm{P}}$, while for large $q$ the surface term should take over.

In Figures 2 and 1, we represent the simulation results for the mean square amplitude of the peristaltic-protrusion mode in terms of $\gamma_{\mathrm{eff}}^{\mathrm{P}}(q) \equiv U^{\mathrm{P}}(q) / q^{2}=k T /\left(q^{2}\left\langle\left|\hat{\xi}_{q}^{\mathrm{P}}\right|^{2}\right\rangle A_{0}\right)$. These can be directly compared with the description of the undulatory mode in terms of $\gamma^{\mathrm{U}}(q)$ and $\gamma^{\mathrm{CU}}(q)$. At low $q$, this effective $\mathrm{P}$ surface tension diverges as $\sim u_{0}^{\mathrm{P}} / q^{2}$, reflecting the description of the bulk contribution as a surface term. The simplest physical description to cover the full range of wavevectors would be

$$
\gamma_{\mathrm{eff}}^{\mathrm{P}}(q) \equiv \frac{k_{\mathrm{B}} T}{q^{2}\left\langle\left|\frac{\hat{\xi}_{q}^{\mathrm{low}}-\hat{\xi}_{q}^{\mathrm{up}}}{2}\right|^{2}\right\rangle A_{0}} \approx \frac{u_{0}^{\mathrm{P}}}{q^{2}}+\gamma_{0}^{\mathrm{P}}+\kappa^{\mathrm{P}} q^{2},
$$

which is precisely the expression used in Refs. 23 and 6, to describe the peristaltic mode.

Our simulation results show that expression (12) is qualitatively correct in the low (dashed-dotted line) regime but it does not provide a good quantitative fit in the intermediate region, $q \sigma \approx 1.3$, where both the bulk and surface contributions are relevant. In our opinion, the main problem is connected to the peculiar nature of the interior of the bilayer membrane made of flexible aliphatic chains anchored to the external phosphate groups. For long wavelengths, the peristaltic mode imposes that these chains must be locally compressed or expanded, since the anchoring to the heads prevents the tails from filling a region and emptying other adjacent regions. Therefore, the bulk contribution $u^{\mathrm{P}}(q)$ comes from a solid-like elasticity of the hydrocarbon chains (see dashed-dotted line in Fig. 1). However, for shorter wavelength $(q \sigma \gtrsim 1)$ the interior of the membrane behaves as a hydrocarbon fluid, because the tail of a chain anchored in the compressed regions may reach the adjacent expanded regions. Therefore, the local deformation of the membrane thickness has a much smaller elastic contribution, $u^{\mathrm{P}}(q) \ll u_{0}^{\mathrm{P}}$, and it should become completely soft for large wavevectors, leaving surface-protrusion contribution $\gamma^{\mathrm{P}}(q)$ as the only relevant term (dotted line in Fig. 1). From the analysis of our simulations results, we find that the transition from the elastic solid to the fluid behavior is very accurately described by a gaussian term $u^{\mathrm{P}}(q)=u_{0}^{\mathrm{P}} \exp \left(-\left(q / q_{\mathrm{el}}^{\mathrm{P}}\right)^{2}\right)$ (see dashed dark (red) line in Fig. 1).

In the large $q$ regime, where the peristaltic-protrusion mode is fully controlled by the protrusion surface term, our simulation results show a deviation from the simplest form $\gamma^{\mathrm{P}}(q)=\gamma_{0}^{\mathrm{P}}+\kappa^{\mathrm{P}} q^{2}$, similar to that observed for the $\mathrm{CU}$ mode. For larger $q \sigma>2.5 \gamma^{\mathrm{P}}(q)$ features a rapid increase (i.e., a rapid decay of $\left.\left\langle\left|\hat{\xi}_{q}^{\mathrm{P}}\right|^{2}\right\rangle\right)$, however, as we can see in the supplementary material ${ }^{58}$ this behavior strongly depends on the procedure used to define the intrinsic surface and therefore we will not analyze this range of $q$. The final expression that we will use in order to represent the peristaltic-protrusion mode (continuous lines in Figures 2 and 1) is

$$
\begin{aligned}
\gamma_{\mathrm{eff}}^{\mathrm{P}}(q) & \equiv \frac{U^{\mathrm{P}}(q)}{q^{2}}=\frac{u^{\mathrm{P}}(q)}{q^{2}}+\gamma^{\mathrm{P}}(q) \\
& =\frac{u_{0}^{\mathrm{P}}}{q^{2}} \exp \left[-\left(\frac{q}{q_{\mathrm{el}}^{\mathrm{P}}}\right)^{2}\right]+\gamma_{0}^{\mathrm{P}}+\kappa^{\mathrm{P}} q^{2}
\end{aligned}
$$

and the best fitting parameters of the fluctuations to these equations are given in Table II.

\section{E. The undulatory and monolayers fluctuations}

A simple mechanical analogy of our approach is presented in Fig. 3. The thermal fluctuations of the mean surface correspond to those of an arrangement of springs in series for the $\mathrm{CU}$ and $\mathrm{P}$ modes, so that, when their mean square fluctuations are added,

$$
\left\langle\left|\hat{\xi}_{q}^{\mathrm{U}}\right|^{2}\right\rangle=\left\langle\left|\hat{\xi}_{q}^{\mathrm{CU}}\right|^{2}\right\rangle+\left\langle\left|\hat{\xi}_{q}^{\mathrm{P}}\right|^{2}\right\rangle,
$$




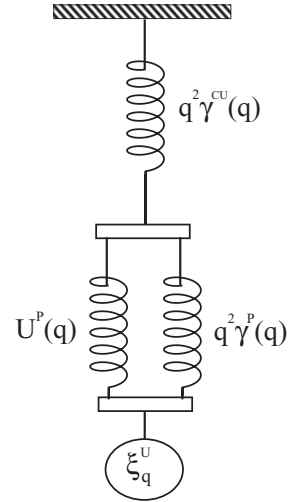

FIG. 3. Sketch for the elastic behavior of the mean surface undulations $\xi_{q}^{U}$ in a bilayer membrane. The complex $q$-dependence of the effective spring constant $q^{2} \gamma^{\mathrm{U}}(q)$ (open circles in Figs. 1-3) results from the combination of three elementary springs. Those with surface character become very soft for small wavevectors, with spring constants $q^{2} \gamma^{x}(q)$, and different $q$-dependent surface tensions $(x=\mathrm{CU}, \mathrm{P}$, and $\mathrm{U})$ for the coupled undulatory, uncoupled protrusions, and mean undulatory fluctuations. The peristaltic fluctuations in the membrane thickness are bulk-like, so that their effective spring constant $U^{P}(q)$ is stiff for $q=0$ and it becomes weaker for increasing $q$.

an effective force constant $\sim q^{2} \gamma^{\mathrm{U}}(q)$ results, which can be calculated from

$$
\frac{1}{\gamma^{\mathrm{U}}(q)}=\frac{1}{\gamma^{\mathrm{CU}}(q)}+\frac{1}{\gamma_{\mathrm{eff}}^{\mathrm{P}}(q)}
$$

For very dissimilar spring constants, the elasticity constant is that of the weakest spring in the series.

The bulk-peristaltic and the surface-protrusion contributions to the $\mathrm{P}$ mode act as parallel springs, adding their force constants to $u^{\mathrm{P}}(q)+q^{2} \gamma^{\mathrm{P}}(q)$, so that for very different values of the two terms the strongest spring dominates the $\mathrm{P}$ mode. The effective spring for the fluctuations of the mean surface is then given by

$$
q^{2} \gamma^{\mathrm{U}}(q)=\left[\frac{1}{q^{2} \gamma^{\mathrm{CU}}(q)}+\frac{1}{u^{\mathrm{P}}(q)+q^{2} \gamma^{\mathrm{P}}(q)}\right]^{-1},
$$

and its dependence with $q$ reflects the transition from the undulatory regime, when the peristaltic spring is too rigid to be relevant, to the protrusion mode when $u^{\mathrm{P}}(q)$ vanishes and $\gamma^{\mathrm{CU}}(q)$ diverges (i.e., $\left\langle\hat{\xi}^{\mathrm{up}} \hat{\xi}^{\text {low* } *}\right\rangle \approx 0$ ).

For the fluctuations of one of the monolayers, we have

$$
\left\langle\left|\hat{\xi}_{q}^{\mathrm{m}}\right|^{2}\right\rangle=\left\langle\left|\hat{\xi}_{q}^{\mathrm{CU}}\right|^{2}\right\rangle+2\left\langle\left|\hat{\xi}_{q}^{\mathrm{P}}\right|^{2}\right\rangle
$$

so that the elementary springs of the $\mathrm{P}$ mode enter with half their values in $\gamma^{\mathrm{U}}(q)$,

$$
q^{2} \gamma^{\mathrm{m}}(q)=\left[\frac{1}{q^{2} \gamma^{\mathrm{CU}}(q)}+\frac{2}{u^{\mathrm{P}}(q)+q^{2} \gamma^{\mathrm{P}}(q)}\right]^{-1} .
$$

In the intermediate range, $0.5 \mathrm{~nm} \lesssim q \lesssim 1.5 \mathrm{~nm}$, when the three springs have similar force constants, the $\mathrm{U}$ and $\mathrm{m}$ data represent a mixed regime in which the fluctuations of the two edges in the bilayer are partially coupled. We note that if we had to estimate the bending modulus $\kappa$ from the undulations of the mean surface (i.e., $\gamma^{\mathrm{U}}(q)$ ) or from those of one of the monolayers (i.e., $\gamma^{\mathrm{m}}(q)$ ), using only that range of $q$ in Fig. 1, we would have very large errors.
The different information obtained from by $\gamma^{\mathrm{U}}(q)$ and $\gamma^{\mathrm{m}}(q)$ could be used to extract quantitative information on the "elementary springs" describing the membrane elastic behavior. Similarly, different experimental techniques, X-ray and neutron scattering, which are sensitive to different aspects of the bilayer molecular structure, could be used to separate the contributions of the elementary springs. In computer simulations, which provide full access to the molecular positions, the most efficient and robust procedure to do so is to sample separately the fluctuations of the upper and lower layers (see Fig. 3), i.e., the $\mathrm{CU}$ and $\mathrm{P}$ modes used in this work.

\section{RESULTS AND DISCUSSION}

\section{A. The coupled undulatory and peristaltic-protrusion modes}

The simulation results for the $\mathrm{CU}$ and $\mathrm{P}$ modes in Figures 1 and 2 can be accurately fitted to Eqs. (11) and (13). The value of $\gamma_{0}$ that gives the best fit of the $\mathrm{MD} \gamma^{\mathrm{CU}}(q)$ results to Eq. (11) is always close to the macroscopic surface tension imposed in the simulations, although with this method we cannot resolve variations in the surface tension smaller than $0.5 \mathrm{mN} / \mathrm{m}$. The results in Table II were obtained assuming that $\gamma_{0}$ takes exactly the macroscopic value. Therefore, the only parameter that is relevant to the fit of the $C U$ mode at $q \sigma \lesssim 0.6$ is the bending modulus, while for larger $q$ our empirical description for the rapid increase of $\gamma^{\mathrm{CU}}(q)$ becomes important. Advancing the discussion in Subsection IV B, we note that for the tensionless membranes a more sensitive representation of the low $q$ fluctuations may be required to determine $\gamma_{0}$. The expansions of (16) and (18) at low $q$ imply that the bending modulus from the fit $\gamma^{\mathrm{x}}(q)=\gamma_{o}+\kappa^{\mathrm{x}} q^{2}+\cdots$, depends on the representation of the undulatory mode,

$$
\kappa^{\mathrm{U}}=\kappa-\frac{\gamma_{o}^{2}}{u_{0}^{\mathrm{P}}} \quad \text { and } \quad \kappa^{\mathrm{m}}=\kappa-2 \frac{\gamma_{\mathrm{o}}^{2}}{\mathrm{u}_{0}^{\mathrm{P}}},
$$

where $\kappa$ is the $\mathrm{CU}$ result, and $u_{0}^{\mathrm{P}}$ the $q=0$ term of the peristaltic mode. Hence, the bending modulus is a well-defined macroscopic parameter only when the membrane is in the tensionless state, whereas for membranes under tension the bending depends on the surface used to quantify the fluctuations. Nevertheless, in typical biological membranes this dependence is usually minor. As a matter of fact in our POPC simulations, even at the highest surface tension $\gamma_{o}=27.3$ $\mathrm{mN} / \mathrm{m}$, we find $\beta \gamma_{o}^{2} / u_{0}^{\mathrm{P}}=0.9 \ll \beta \kappa \approx 21$, so that the difference $\kappa^{\mathrm{x}}-\kappa$ is within the accuracy of our method. In practice, the main difference between the analysis of $\gamma^{\mathrm{CU}}(q)$ and that of $\gamma^{\mathrm{U}}(q)$ is that the estimation of the $\kappa$ from the latter requires the analysis of larger systems to avoid the mixing with the protrusion.

The result $\beta \kappa \approx 21$, obtained from the $\mathrm{CU}$ mode in our POPC model, does not depend significantly on the membrane surface tension (from $\gamma_{0}=0$ to $27.3 \mathrm{mN} / \mathrm{m}$ ), although the area per molecule $A_{\text {POPC }}$ (Table I) and mean thickness of the bilayer (Table II) $d=\left\langle\hat{\xi}_{0}^{\text {up }}-\hat{\xi}_{0}^{\text {low }}\right\rangle$ are modified under these conditions, so that the density of the aliphatic chains in the bilayers is approximately constant. That result for $\kappa$ is close to available experimental values for POPC, $\beta \kappa=24 \pm 0.3$, 
obtained with X-rays ${ }^{60}$ and confirmed by the analysis of fluctuations in vesicles. ${ }^{61}$ The wavevector cutoff is close, although lower, to the empirical guess $q_{\mathrm{u}}^{\mathrm{CU}} \approx 2 \pi / d$. Within the error bars it is not possible to establish a clear dependence of $q_{\mathrm{u}}^{\mathrm{CU}}$ with $\gamma_{0}$.

For the tensionless DPPC membrane, we have obtained a bending modulus of $\beta \kappa=23.0 \pm 1.5$, which agrees with the experimental expectations. ${ }^{62,63}$ We note that in Ref. 63 values of the order of $\beta \kappa=40$ were reported near the liquidcrystal-gel phase transition. These values can be compared with those obtained by different laboratories using the present coarse grained model. In the seminal paper using the MARTINI force-field, Marrink et al. ${ }^{64}$ used the usual undulatory approach to quantify the bending modulus of DPPC. They obtained a very low value, $\beta \kappa=9.6$. Later, Otter and Shkulipa ${ }^{65}$ used the same procedure but increased the lateral size of the membrane. Their value was much higher, $\beta \kappa=19.3$, which is closer to the value obtained by us in this work. Shkulipa ${ }^{66}$ also employed a finite size scaling approach, where the bending modulus is computed from the slope of the compressibility modulus versus membrane size. The value using this method turned up to higher $\beta \kappa=27.0,{ }^{66}$ again closer to our result. Our bending modulus is much lower than the value reported by Watson et al., ${ }^{31}$ $\beta \kappa=36$. These authors quantified the bending modulus from the fluctuations of the molecular orientations, rather than from the positions of the molecular heads as we do here, but both approaches have in common than they filter out the protrusions of individual molecules at large $q$ values, so that the mean square amplitude of the (so defined) undulations decreases very rapidly in the mesoscopic range for $q \gtrsim 0.5 \mathrm{~nm}$. The same effect can be achieved with our method, because the head groups on the opposite sides of the bilayer are decoupled for $q \gtrsim 2 \pi / d$. With the surface curvature defined through the molecular orientations, ${ }^{31}$ the flexible aliphatic chains sample the internal structure of the membrane, so that their orientations give a smeared view of the local orientation. As in the softening of the peristaltic mode at high $q$, the molecular orientations do not keep track of the surface undulations at scales shorter than the molecular length $q \sigma \gtrsim 1$. Both methods could be applied to relatively small systems, because they avoid the entanglement of undulations and protrusions, and they should give the same result for the bending modulus at $\gamma_{0}=0$. The possible origin of the discrepancy is analyzed in Subsection IV B.

In our approach, the membrane elasticity parameters are obtained from the fitting of $\gamma_{\mathrm{eff}}^{\mathrm{P}}(q)$ to Eq. (13). For our POPC model, we get values for the bulk modulus associated to the peristaltic deformations from $\beta u_{0}^{\mathrm{P}}=26.0 \mathrm{~nm}^{-4}$ for tensionless membranes, to $20.0 \mathrm{~nm}^{-4}$ for $\gamma_{0}=27.3 \mathrm{mN} / \mathrm{m}$. It is well known that this elastic constant strongly depends on the chemical nature of the amphiphilic molecule. Our results for POPC membranes may be relatively high, due to the coarsegrained description used here for the hydrocarbon tails. Lower values, $\beta u_{0}^{\mathrm{P}}=14.9 \pm 5 \mathrm{~nm}^{-4}$, have been reported for glycerolmonoolein bilayer membranes, ${ }^{6}$ and a surprisingly low value (ten times smaller) has been estimated for the DPPC membrane. ${ }^{23}$ In contrast, our results give very similar values for $\beta u_{0}^{\mathrm{P}}$ with the (similar) coarse grained models for POPC and DPPC. This is not surprising considering the similarities of these phospholipids in the coarse grained description.

The values $q_{\mathrm{el}}^{\mathrm{P}} \approx 0.8 / \sigma$ (Table II) indicate that the softening in the peristaltic mode appears for wavelengths $2 \pi / q$ $\gtrsim 6 \mathrm{~nm}$, i.e., twice the maximum length of the aliphatic chain in the lipid molecules. This result agrees with our qualitative interpretation of this softening, as due to the transition from the elastic solid to the fluid behavior of the phospholipids hydrocarbon tails. The parameter $\gamma_{0}^{\mathrm{P}}$ in Eq. (13) has to be interpreted as twice the surface tension in a phospholipid monolayer, and it controls the uncoupled protrusions on the two sides of the bilayer membrane. Our result $\gamma_{0}^{\mathrm{P}} \approx 13.0\left(\beta \sigma^{2}\right)^{-1}=86 \mathrm{mN} / \mathrm{m}$ for the tensionless bilayer is within the range of reported values in the literature, from 30 to $180 \mathrm{mN} / \mathrm{m}^{21,23,26,38}$ Brandt et al. ${ }^{32}$ have suggested that, due to the high value of the $\gamma_{0}^{\mathrm{P}}$, the contribution of the protrusions to the undulatory mode is negligible. However, we have found that although its contribution is weak, it is necessary to include a surface tension protrusion term in order to accurately describe the intermediate q-range of the fluctuation spectrum. Note that Brandt et al. ${ }^{32}$ assumed a bending like behavior of the undulatory mode over the entire range of $q$, while we have found that the coupled undulatory mode diverges to intermediate $q$. This could have an effect on the results obtained for the intermediate q-range. Also, Brandt et al.'s ${ }^{32}$ unconventional static number density structure factor, dominates at large $q$ vectors, and it includes contributions that correspond to our peristaltic-protrusion mode.

Finally, we comment on the parameters that describe the behavior of the molecular protrusions at large $q$, i.e., beyond the range where $\gamma^{\mathrm{P}}(q) \approx \gamma_{0}^{\mathrm{P}}$. The $\mathrm{P}$ mode bending modulus $\kappa^{\mathrm{P}}$, obtained from the fit of the simulation results to Eq. (13), is almost residual, being much lower than the (macroscopic) undulatory modulus $\kappa$. Moreover, $\kappa^{\mathrm{P}}$ depends on the procedure used to define the intrinsic surface. We must note that the only physical reality behind the instantaneous shape $\xi(\mathbf{R})$ is the discrete set of pivots, i.e., the lipid phosphate groups. In the supplementary material, ${ }^{58}$ we investigate the dependence of our results with the approach used to construct the nominal surface that interpolates between these pivots. We have found that, contrary to the robustness of the other parameters, the protrusion bending modulus $\kappa^{\mathrm{P}}$ is strongly affected by the approach used to construct the nominal surface. Its value is certainly much smaller than $\kappa$.

\section{B. The low q behavior of the fluctuation spectrum}

The traditional approach to estimate the bending modulus of a free membrane $\left(\gamma_{o}=0\right)$ uses the function $\Xi^{\mathrm{x}}(q)=q^{4}\left\langle\left|\hat{\xi}_{q}^{\mathrm{x}}\right|^{2}\right\rangle A_{0}^{-1}=k T q^{2} / \gamma^{\mathrm{x}}(q)$, which converges in the $q=0$ limit to $\Xi^{\mathrm{x}}(q) \approx(\beta \kappa)^{-1}+\mathcal{O}\left(q^{2}\right)$. This function is very sensitive to the low- $q$ undulations, much more sensitive than our direct fit to $\gamma^{\mathrm{CU}}(q)$ to (11). The latter is on the other hand more sensitive to the undulations in the intermediate $q$ range, which is important to disentangle the different modes. Our simulation results for POPC (Fig. 4) and DPPC (Fig. 5) show a low- $q$ structure of $\Xi^{\mathrm{CU}}(q)$, which features a maximum at $q_{\max } \approx 0.7 \mathrm{~nm}^{-1}$. This maximum disappears, $\Xi^{\mathrm{CU}}(q)$ does 


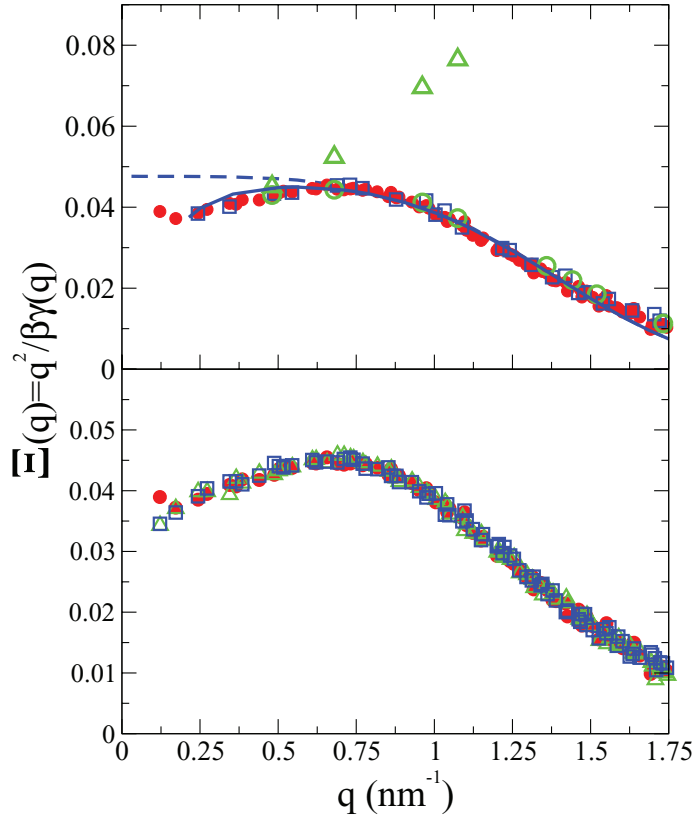

FIG. 4. The height coupled undulatory fluctuations $\Xi^{\mathrm{CU}}(q)$ $=q^{4}\left\langle\hat{\xi}_{q}^{\text {low }} \hat{\xi}_{q}^{u p *}\right\rangle=\frac{q^{2}}{\beta \gamma^{\mathrm{CU}}(q) A_{0}}$. (Top panel) Results for the tensionless POPC membrane obtained with different lateral size $L_{x}$ of the simulation box. The empty squares (blue) are the size used all over this work $\left(L_{x} \approx 27 \mathrm{~nm}\right)$; the full (red) circles have twice linear size $\left(L_{x} \approx 50.2 \mathrm{~nm}\right)$, and the empty (green) circles half linear size $\left(L_{x} \approx 12.7 \mathrm{~nm}\right)$, the POPC density is the same at the three simulations. The triangles give the undulatory results $\Xi^{\mathrm{U}}(q)$ for the smallest system with the usual representation of the undulatory mode by the mean surface. The dashed line is the corresponding fit of $\gamma^{\mathrm{CU}}(q)$ results to our parametrized equation (11), and the full line is the fit of $\Xi^{\mathrm{CU}}(q)$ results to our parametrized equation (20) assuming a non-zero value of the fluctuating surface tension. (Bottom panel) Simulations results for the $L_{x} \approx 50.2 \mathrm{~nm}$ POPC membrane in different ensembles. Circles (red): the $\left(N_{P h o s}, \gamma_{0}=0\right)$ shown in the top panel with the barostat used all over this work; triangles (green): $\left(N_{P h o s}, \gamma_{0}=0\right)^{*}$ with the weak coupling barostat; squares (blue): ( $\left.N_{P h o s}, A_{0}\right)$ with projected lateral area fixed to the mean value obtained with null surface tension.

not decrease to low $q$, if we evaluate $\Xi^{\mathrm{CU}}(q)=q^{2}\left(\beta \gamma^{\mathrm{CU}}(q)\right)^{-1}$ with the analytical fits of $\gamma^{\mathrm{CU}}(q)$ to Eq. (11) assuming $\gamma_{0}$ $=0$, obtained in Sec. IV A. These discrepancies had very little weight in the direct fit to $\gamma^{\mathrm{CU}}(q)$, but it becomes obvious in the new representation $\Xi^{\mathrm{CU}}(q)$. Our previous estimates for $\beta \kappa$ are very close to the inverse of $\Xi^{\mathrm{CU}}\left(q_{\max }\right) \approx 0.048$ for POPC, and 0.044 for DPPC. With the usual definition of the undulatory term, the function $\Xi^{\mathrm{U}}(q)$ approaches our $\Xi^{\mathrm{CU}}(q)$ for low $q$, but instead of reaching a maximum it grows monotonically over the whole range of wavevectors. That effect is produced by the mixing with the protrusions part of the peristaltic mode that pushes $\gamma^{\mathrm{U}}(q)$ below the quadratic expansion $\gamma_{o}+\kappa q^{2}$ as shown in Fig. 1.

We have performed several tests to explore the origin of the non-monotonic behavior of $\Xi^{\mathrm{CU}}(q)$. The upper panel in Fig. 4 compares the results for three system sizes, $N_{\text {Phos }}$ $=256,1000$, and 4000 phospholipids per layer; and box lateral sizes from $L_{x} \approx 12.5 \mathrm{~nm}$ to $54 \mathrm{~nm}$, to ensure the $\gamma_{o}=0$ condition. The lower panel compares the results with two different barostats (strong and weak coupling with the phospholipids), and also those obtained in a simulation with fixed $\left(N_{\text {Phos }}, A_{0}\right)$, choosing the projected area equal to the

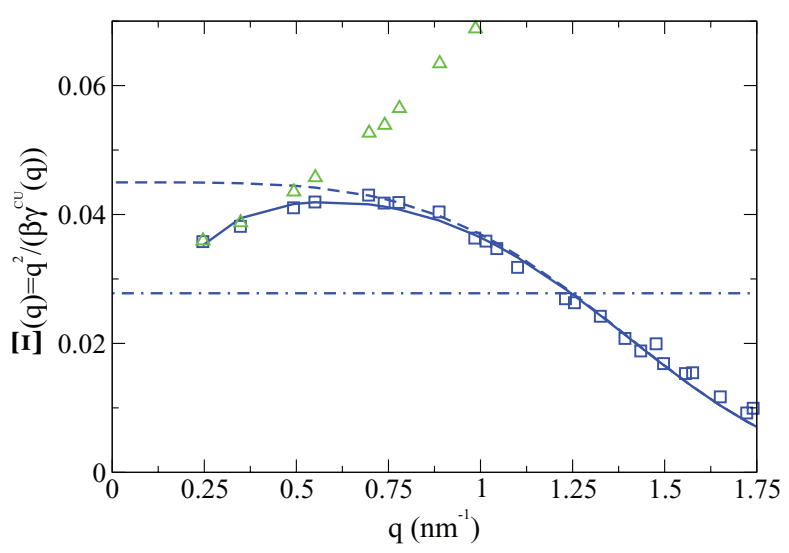

FIG. 5. The height coupled undulatory fluctuations multiplied by $q^{4}$,e.i, $\Xi^{\mathrm{CU}}(q)=q^{4}\left\langle\hat{\xi}_{q}^{\text {low }} \hat{\xi}_{q}^{u p *}\right\rangle=\frac{q^{2}}{\beta \gamma^{\mathrm{CU}}(q) A_{0}}$, of the tensionless DPPC membrane. The symbols squares (blue) are the simulation results; squares (blue) the coupled undulatory $\mathrm{CU}$ mode, and the triangles (green) the undulatory $\mathrm{U}$ mode, $\Xi^{\mathrm{U}}(q)$. The full line is the fit of the $\mathrm{CU}$ results to our parametrized equation (20) assuming a non-zero value of the fluctuating surface tension, and the dashed line represents the same fit imposing $\gamma_{0}=0.0$. The dashed-dotted line is the bending value predicted by Watson et al. ${ }^{31}$

mean value $\left\langle A_{0}\right\rangle_{\gamma_{0}}$ obtained in the simulations with $\gamma_{0}=0$. The dynamics of the corrugations with $q \sigma \lesssim 0.5 \mathrm{~nm}$ is extremely slow. We thus allowed for enough sampling time to get good statistics for $\left\langle\hat{\xi}_{q}^{l o w} \hat{\xi}_{q}^{u p *}\right\rangle$. The results clearly show that the peculiar shape of $\Xi^{\mathrm{CU}}(q)$ (see Figure 4) at low $q$ is not a spurious artifact of the simulations.

For membranes under tension $\left(\gamma_{0}>0\right)$, the presence of a maximum in $\Xi^{\mathrm{CU}}(q)$ is accurately described with our fitting formula (11), since $\Xi(q)=k T q^{2} / \gamma(q) \approx q^{2} /\left(\beta \gamma_{0}+\beta \kappa q^{2}\right.$ $+\cdots)$. In that case, the estimation of the bending modulus has to be done from the simulations results for $q \approx q_{\max }$, rather than from the $q=0$ limit, since $\Xi^{\mathrm{CU}}(q) \approx q^{2} /\left(\beta \gamma_{0}\right)$ $\ll(\beta \kappa)^{-1}$ for $q \ll \sqrt{\gamma_{0} / \kappa}$. Even for the largest $\gamma_{0}$ used in our POPC simulations, the approach to the quadratic low- $q$ behavior of $\Xi(q)$ could only be observed in extremely large systems $\left(L_{x} \gtrsim 200 \mathrm{~nm}\right)$, and the required size would grow as $\sim \gamma_{0}^{-1 / 2}$ as the surface tension is reduced. At the inflection point separating the minimum at $q=0$ and the maximum at $q \approx 0.7$ $\mathrm{nm}^{-1}$, we would observe $\Xi(q)^{-1} \approx 4 \beta \kappa$, independently of the value of $\gamma_{0}$. For example, in the bottom panel of Fig. 6 the arrow marks this inflexion point for a tensed POPC membrane. For a membrane under a small tension, the extrapolation of $\Xi(q)$ to $q=0$ would produce a gross overestimation $\kappa$, if the membrane is assumed to be in the tensionless state.

It has been pointed by Barbetta and Fournier ${ }^{18}$ that the fluctuations of a free membrane lead to a small effective surface tension, $\gamma_{\text {fluct }} \geq 0$, even if the simulation is performed at zero surface tension, $\gamma_{0}=0$. If we fit our simulation results to the form

$$
\Xi^{\mathrm{CU}}(q) \equiv \frac{q^{2}}{\gamma^{\mathrm{CU}}(q)}=\frac{q^{2}}{\gamma_{\text {fluct }}+\kappa q^{2}\left[1+\left(\frac{q}{q_{\mathrm{u}}^{\mathrm{CU}}}\right)^{\alpha^{\mathrm{CU}}}\right]},
$$

allowing a residual non-zero value of the surface tension as a free parameter, we get the excellent fits shown by the full lines in Figs. 4 and 5. The best fitting values are $\beta \gamma_{f l u c t} \sigma^{2}=0.43$ 


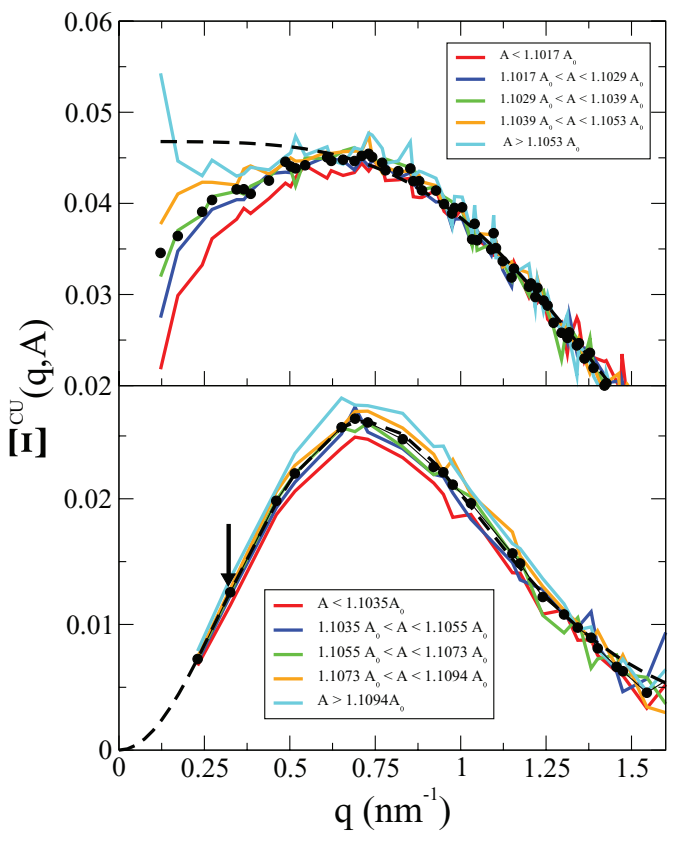

FIG. 6. The height coupled undulatory fluctuations multiplied by $q^{4}$,e.i, $\Xi^{\mathrm{CU}}(q, A)=q^{4}\left\langle\hat{\xi}_{q}^{l o w} \hat{\xi}_{q}^{u p *}\right\rangle$ as function of the real area of the membrane $A$. The symbols show the results without the real area constraint, $\Xi^{\mathrm{CU}}(q)$. The dashed line is the fit of the $\gamma^{\mathrm{CU}}(q)$ simulation results to the parametrized equation (11). (Top panel) POPC membrane with lateral area $A_{0}$ fix and $N_{P h o s}$ $=4000$. (Bottom panel) POPC membrane under tension, $\gamma_{0}=27.3 \mathrm{mN} / \mathrm{m}$, and $N_{\text {Phos }}=1000$. The arrow marks the inflexion point of $\Xi^{\mathrm{CU}}(q)$.

$\left(\gamma_{\text {fluct }}=2.8 \mathrm{mN} / \mathrm{m}\right)$, for DPPC, and $\beta \gamma_{\text {fluct }} \sigma^{2}=0.38\left(\gamma_{\text {fluct }}\right.$ $=2.5 \mathrm{mN} / \mathrm{m}$ ), for POPC. Nevertheless, we find that the best fitting values for $\kappa$ (and also the parameters $q_{\mathrm{u}}^{\mathrm{CU}}, \alpha^{\mathrm{CU}}$ ) are very close to those obtained in Subsection IV A, with direct fits to $\gamma^{\mathrm{CU}}(q)$ under the restriction $\gamma_{0}=\gamma_{\text {fluct }}=0$. The robustness of the bending modulus to small changes in $\gamma_{\text {fluct }}$ gives further support to our estimate of $\beta \kappa=23.0 \pm 1.5$ for the bending modulus of the tensionless DPPC membrane.

We turn now to the core of this question: whether the surface tension imposed by the barostat on a membrane $\gamma_{0}$ is equal or not to that estimated through the analysis of the microscopic fluctuations. Discussion about this problem can be found in previous works, which either suggests these surface tensions are equal ${ }^{15-17}$ or different. ${ }^{18-20}$ The barostat surface tension $\gamma_{0}$ is the conjugated variable to the projected area $A_{0}$ of the simulation box, while $\gamma_{\text {fuct }}$, obtained from the membrane fluctuations, is related to the true area of the corrugated membrane $A$. This is an important difference when $\gamma_{0}=0$. The prediction from Helfrich surface hamiltonian is that for $\gamma_{0}>0$ the difference $\langle A\rangle \approx A_{0}[1+\log (1$ $\left.\left.+\kappa q_{u}^{2} / \gamma_{0}\right) /(4 \pi \beta \kappa)\right]$, with $q_{u} \sim 2 \pi / \sigma$, with a proportionality factor roughly independent of the system size, so that $A_{0}$ or $A$ may be used indistinctly as the thermodynamic conjugate variable to the surface tension $\gamma_{0}$. However, as pointed by Schmid ${ }^{15}$ for a tensionless membrane the conjugated ensembles $\left(N_{\text {Phos }}, \gamma_{0}=0\right)$ and $\left(N_{\text {Phos }}, A_{0}\right)$ are not representative of a truly free membrane that fluctuates in the $\left(N_{P h o s}, A\right)$ ensemble.

To further analyze this point, we have run simulations where the fluctuations of the instantaneous triangulated surface area (within a narrow range $1.10 \lesssim A / A_{0} \lesssim 1.12$ ) were an-

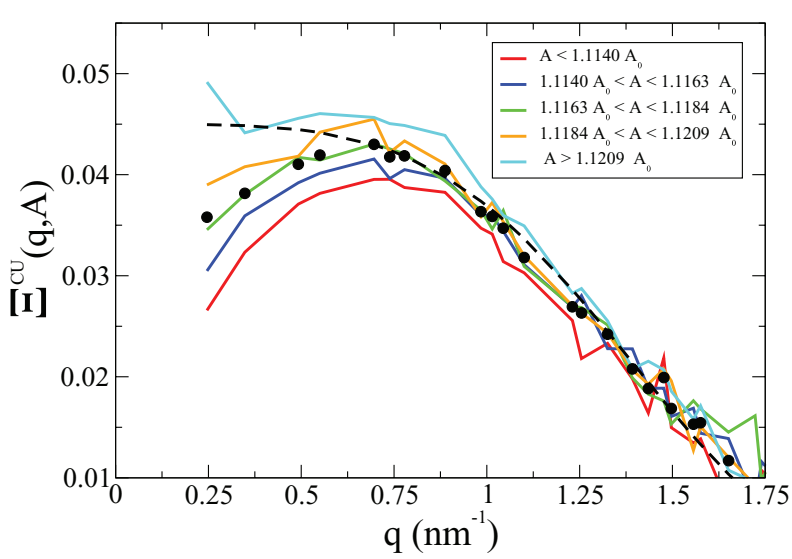

FIG. 7. The height coupled undulatory fluctuations multiplied by $q^{4}$,e.i, $\Xi^{\mathrm{CU}}(q, A)=q^{4}\left\langle\hat{\xi}_{q}^{\text {low }} \hat{\xi}_{q}^{u p *}\right\rangle$, as function of the real area of the membrane $A$, of the tensionless DPPC membrane with 1024 phospholipids per layer. The symbols show the results without the real area constraint, $\left(\Xi^{\mathrm{CU}}(q)\right)$, showed in Fig. 5. The dashed line is the fit of the $\gamma^{\mathrm{CU}}(q)$ simulation results to the parametrized equation (11).

alyzed. The membrane configurations were split into five subensembles, and the function $\Xi^{\mathrm{CU}}(q ; A)$ was computed for each ensemble. The top panel in Fig. 6 shows $\Xi^{\mathrm{CU}}(q ; A)$ for the POPC membrane with fixed projected lateral area $A_{0}$, whereas Fig. 7 corresponds to DPPC tensionless membrane, i.e., $\gamma_{0}=0$. Both phospholipid models give qualitatively similar results regardless of the simulation trajectory used. For $q \gtrsim q_{\max } \approx 0.75 \mathrm{~nm}^{-1}$, all the area sub-ensembles give the same results. Adding all the sub-ensembles together reduces the noise in the results only, showing they are independent. However, for $q \lesssim q_{\max }$ the results of the sub-ensembles feature large deviations. The configurations with smaller area give a higher slope in $\Xi(q)$. When these results are fitted to Eq. (20), we find that these configurations predict larger values of $\gamma_{\text {fluct }}$. The ensemble corresponding to the most corrugated membranes, i.e., those in the upper range of $A$, give $\Xi(q) \approx \Xi\left(q_{\max }\right)$. Although the result features some noise they are compatible with the tensionless state, i.e., $\gamma_{\text {fluct }} \approx \gamma_{0}=0$. These are the configurations that provide the closer representation of a free-standing tensionless membrane.

The bottom panel in Fig. 6 shows that for membranes under tension $\left(\gamma_{0}=27.3 \mathrm{mN} / \mathrm{m}\right.$ in this case) the results for $\Xi^{\mathrm{CU}}(q ; A)$ are very similar for the sub-ensembles. The effective values of $\gamma_{\text {fluct }}$ that result from the fitting to Eq. (20) have smaller and more symmetric fluctuations around $\gamma_{0}$. Our results agree with the work by Neder $e t a l .^{22}$ The practical consequence of this observation is that the fluctuations of these membranes under tension can be quantified with computer simulations at fixed $\left(N_{\text {Phos }}, \gamma_{0}\right)$ or fixed $\left(N_{\text {Phos }}, A_{0}\right)$, as well as in sub-ensembles with fixed ( $\left.N_{\text {Phos }}, A\right)$. In all these cases, the fluctuations agree with each other. Notice that our estimations of the bending modulus in each $A$ sub-ensemble would result in very similar values too, $\beta \kappa \approx 21 \pm 1$ for POPC, and $\beta \kappa \approx 23 \pm 1$ for DPPC, which are in excellent agreement with our previous results, which were obtained from a direct fit to $\gamma^{\mathrm{CU}}(q)$. It is expected that $\kappa$ changes very little with $\gamma_{\text {fluct }}$, since (for POPC) we have found that the value of $\kappa$ is 
insensitive to much larger changes of the externally imposed tension $\gamma_{0}$.

To summarize, a consistent estimation of $\kappa$ can be made using different approaches. First, by allowing for a small effective surface tension created by the fluctuations $\left(\gamma_{\text {fluct }}\right.$ $\lesssim 3 \mathrm{mN} / \mathrm{m}$ ). Second, by using simulation results with a small surface tension $0<\gamma_{0} \lesssim 10 \mathrm{mN} / \mathrm{m}$ and third, by sorting the configurations into different sub-ensembles, which feature different fluctuating areas, $A$. In contrast, the analysis of simulation data obtained from trajectories corresponding to $\gamma_{0}$ $=0$, or for fixed $A_{0}$, lead to a strong overestimation of the bending modulus, when the data at low $q$-vectors are extrapolated to $q \rightarrow 0$ and then $\beta \kappa=\Xi(q \rightarrow 0)^{-1}$ used to estimate the bending modulus. Using this approach, we find $\beta \kappa \approx 33$ for POPC and $\beta \kappa \approx 36$ for DPPC. Interestingly, this latter value is similar in magnitude to the one reported by Watson et al.,$^{30,31}$ using the same coarse-grained model for DPPC, and similar size for the bilayer, although in that work a different characterization of the membrane corrugations, in terms of the molecular orientations, was used. The results presented by these authors are restricted to the $\left(N_{\text {phos }}, \gamma_{0}=0\right)$ ensemble, and in their analysis they do not allow for any effective $\gamma_{\text {fluct }}$ $>0$ associated to the membrane fluctuations, neither do they take into account the difference between the thermodynamic control over the projected and the real areas. We suggest that the different treatment of the fluctuation of $A$ used in different experimental techniques, $\mathrm{x}$-ray scattering, analysis of thermal fluctuations, or pipette aspiration techniques, could explain why the experimental estimates of $\kappa$ for the same membrane can vary sometimes by up to $\pm 10 k_{B}$ T units. ${ }^{59,62}$ The small dependence of bending with the surface tension applied that we have obtained in this work, opens the possibility to measure the bending using a membrane under a controlled small tension, hence reducing the fluctuations of $A$.

\section{CONCLUDING REMARKS}

The long wavelength undulations of a bilayer membrane are determined by the bending modulus $\kappa$ and the surface tension $\gamma_{0}$, the two parameters in the Helfrich free energy functional that define the membrane shape $z=\xi(\mathbf{R})$. However, over the mesoscopic range $q \sim 1 \mathrm{~nm}^{-1}$ the undulatory mode is entangled with modes associated to the modification of the inner structure of the membrane. The spectrum of fluctuations in the bilayers evolve continuously from the low- $q$ undulations and the peristaltic changes of the bilayer thickness, to the protrusion and tilt of individual molecules. The estimation of $\kappa$, from the analysis of the membrane thermal fluctuations require very large systems and long time trajectories, to enable a good sampling of the very slow fluctuations with characteristic wavelengths $q \lesssim 0.1 \mathrm{~nm}^{-1}$. We have proposed here a new theoretical approach to compute $\kappa$ from the analysis of small size membranes. The recent work by Watson et al. ${ }^{31}$ had the same goal and employed similar approach. The main idea is that coupled undulations may be introduced to disentangle long and short wavelength fluctuation modes. Here, we have chosen the correlation between the head groups in the opposite sides of the bilayer as order parameter. In this way, the whole analysis can be performed from the knowledge of the position of the phospholipid heads. Instead, Watson et al. ${ }^{31}$ employed the orientation of the phospholipid tails to sample the inner structure of the layers.

For tensionless membranes, i.e., $\gamma_{0}=0$, the bending modulus $\kappa$, determined by any of the methods discussed above should agree with the bending obtained through the usual representation of the undulations, namely, by identifying the membrane local shape with the the mean position of the head groups in the two phospholipid monolayers. However, this representation often leads to an undesired mixing of the undulatory modes with peristaltic and protrusion ones, which can only be avoided when considering very small wavevectors, $q$. The use of a correlation filter, either between the two monolayers or between the molecular position and tilt, provides a route to extract a pure undulatory mode. It is important to note that the choice of different intra-membrane correlations used to define the coupled undulations, may have a significant impact on the effectiveness of the filter in eliminating the short wave fluctuations.

When the membrane is under tension, $\gamma_{0}>0$, the situation is slightly different, since the bending modulus cannot be formally defined from the low $q$-limit, as this limit is dominated by the surface tension term. Hence, the value of $\kappa$ as defined in the Helfrich Hamiltonian depends on the precise (molecular) definition of the membrane shape. We find that different, reasonable choices for the membrane shape, result in small changes in the bending, about $\Delta \kappa \lesssim 1 k T$ over typical values $\kappa \gtrsim 20 k T$. That should be considered as an inherent uncertainty in the bending modulus of tensed membranes, $\Delta \kappa \sim \gamma_{0}^{2}$, that only disappears in a free standing membrane.

The simulations at $\gamma_{0}=0$ present a subtle difficulty that we believe is at the heart of the apparent discrepancy between the result $\beta \kappa \approx 36$ reported by Watson et al. ${ }^{31}$ for a coarsegrained model of DPPC, and our results $(\beta \kappa \approx 23 \pm 1)$, which were obtained with similar membrane sizes and simulation conditions, and using the same force-field and phospholipid membrane. We have shown that the observed non-monotonic behavior of $q^{4}\left\langle\left|\hat{\xi}_{q}^{\text {low }} \hat{\xi}_{q}^{\text {up* }}\right|\right\rangle$, instead of the expected flat limit at $q \rightarrow 0$, explains this discrepancy. The deviations for the flat limit result from the peculiarities of the $\left(N_{\text {phosp }}, \gamma_{0}=0\right)$ ensemble as discussed by Refs. 15 and 18. Hence, the direct extrapolation of our simulation data, using our lowest accessible wavevectors, would give a too high estimate of $\kappa$, with values very close to those reported by Watson et al. ${ }^{31} \mathrm{We}$ have shown that the correct bending modulus can be recovered using three different strategies. (1) Allowing for a small effective surface tension associated to the fluctuations ( $\gamma_{\text {fluct }}$ $\lesssim 3 \mathrm{mN} / \mathrm{m}$ ), (2) using simulation data for membranes under a small surface tension $\gamma_{0}>0$, and (3) by sorting the trajectory in sub-ensembles ( $N_{\text {phosp }}, A$ ), according to the actual area of the membrane, rather than the projected area $A_{0}$. Further work is needed to test our approach in conjunction with the method discussed by Watson et al., ${ }^{31}$ so that full agreement between different methods can be established.

The subtleties associated to the simulation of tensionless membranes are relevant in defining their macroscopic behavior, i.e., for fluctuations with $q<0.5 \mathrm{~nm}^{-1}$. In the mesoscopic range $0.5 \mathrm{~nm}^{-1}<q<1.5 \mathrm{~nm}^{-1}$, where $q^{4}\left\langle\left|\hat{\xi}_{q}^{\text {low }} \hat{\xi}_{q}^{\text {up } *}\right|^{2}\right\rangle$, tensionless simulations, i.e., fixed $\gamma_{0}=0$, fixed project area $A_{0}$ 
or fixed real ares $A$ produce equivalent results. The analysis of the fluctuations in this mesoscopic range requires relatively small membranes $\left(L_{x} \lesssim 20 \mathrm{~nm}\right.$ ), for which the dynamics of $\hat{\xi}_{q}$ fluctuations is faster, enabling better statistics with shorter simulations. This is a definite computational advantage, and makes feasible the quantification of the bending modulus of membranes described with atomistic models.

An added advantage of our method is that in addition to the bending modulus it provides detailed information on the internal fluctuations of the membrane down to the molecular size. By using the coordinates of the lipid head-groups, we have quantified the peristaltic modes of the membrane thickness as well as the protrusions of individual molecules. Obviously, the internal structure of a phospholipid bilayer has many other types of motion that could also be explicitly resolved, e.g., the tilt and extension of the aliphatic chains or the local area per molecule. All these molecular fluctuations are present in the simulations, and it is a matter of choice which aspects of the membrane structure are explicitly sampled and which ones are averaged out in the mesoscopic representation of the membrane elasticity. The representation of the mean surface fluctuations in terms of a combination of elementary springs (see Fig. 3), consisting of a coupled undulatory and an uncoupled protrusion and mean undulatory fluctuations, provides a route to rationalize the behavior of the $\gamma^{\mathrm{U}}$ and $\gamma^{m}(q)$ curves. It could also be possible to use a more complex network of springs, to account for other molecular degrees of freedom. When the springs have very different force constant, the effective elasticity is mainly determined by the spring with the largest force constant when the springs are set in parallel, and by the spring with the smallest force constant when the springs are set in series. In the intermediate $q$-range, the spring force constants are nonetheless of the same order. The transition between the coupled to the uncoupled fluctuations of the membrane monolayers corresponds precisely to the intermediate $q$-range.

\section{ACKNOWLEDGMENTS}

We acknowledge the support of the Spanish Ministry of Science and Innovation (Grant No. FIS2010-22047-C05) and the Comunidad Autónoma de Madrid under program MODELICO (Grant No. S2009/ESP-1691). We would also like to acknowledge the Imperial College High Performance Computing Service for providing computational resources and The Royal Society for providing financial support. F.B. would like to thank the Engineering and Physical Sciences Research Council (U.K.) (EPSRC(GB)) for the award of a Leadership Fellowship.

${ }^{1}$ J. Lundbaek, J. Phys. Condens. Matter 18, S1305 (2006).

${ }^{2}$ H. P. Duwe, J. Kaes, and E. Sackman, J. Phys. (France) 51, 945 (1990).

${ }^{3}$ L. Chernomordik, M. Kozlov, and J. Zimmerberg, J. Membr. Biol. 146, 1 (1995).

${ }^{4}$ W. Helfrich, Z. Naturforsch. C 28, 693 (1973).

${ }^{5}$ S. A. Safran, Statistical Thermodynamics of Surfaces, Interfaces, and Membranes (Addison-Wesley, Reading, MA, 1994).

${ }^{6}$ S. Marrink and A. Mark, J. Phys. Chem. B 105, 6122 (2001).

${ }^{7}$ V. Harmandaris and M. Deserno, J. Chem. Phys. 125, 204905 (2006).

${ }^{8}$ G. Gompper and M. Schick, Chem. Phys. Lett. 163, 475 (1989).

${ }^{9}$ M. Matsen and D. Sullivan, Phys. Rev. A 41, 2021 (1990).
${ }^{10}$ J. Halley and A. Kolan, J. Chem. Phys. 88, 3313 (1988).

${ }^{11}$ A. Somoza, E. Chacón, L. Mederos, and P. Tarazona, J. Phys: Condens. Matter 7, 5753 (1995)

${ }^{12}$ G. Gompper and S. Klein, J. Phys. II France 2, 1725 (1992).

${ }^{13}$ E. Chacón, A. Somoza, and P. Tarazona, J. Chem. Phys. 109, 2371 (1998).

${ }^{14}$ M. Hu, P. Diggins, and M. Deserno, J. Chem. Phys. 138, 214110 (2013).

${ }^{15}$ F. Schmid, EPL 95, 28008 (2011).

${ }^{16}$ W. Cai, T. Lubensky, P. Nelson, and T. Powers, J. Phys. II (France) 4, 931 (1994).

${ }^{17}$ O. Farago and P. Pincus, J. Chem. Phys. 120, 2934 (2004).

${ }^{18}$ J. Fournier and C. Barbetta, Phys. Rev. Lett. 100, 078103 (2008).

${ }^{19}$ A. Imparato, J. Chem. Phys. 124, 154714 (2006).

${ }^{20}$ J. Stecki, J. Phys. Chem. B 112, 4246 (2008).

${ }^{21}$ R. Goetz, G. Gompper, and R. Lipowsky, Phys. Rev. Lett. 82, 221 (1999).

${ }^{22}$ J. Neder, B. West, P. Nielaba, and F. Schmid, J. Chem. Phys. 132, 115101 (2010).

${ }^{23}$ E. Lindahl and O. Edholm, Biophys. J. 79, 426 (2000).

${ }^{24}$ R. Lipowsky and S. Grotehans, Europhys. Lett. 23, 599 (1993).

${ }^{25}$ G. Brannigan, L. Lin, and F. Brown, Eur. Biophys. J. 35, 104 (2006).

${ }^{26}$ G. Brannigan and F. Brown, Biophys. J. 90, 1501 (2006).

${ }^{27}$ B. West, F. Brown, and F. Schmid, Biophys. J. 96, 101 (2009).

${ }^{28}$ M. Hamm and M. Kozlov, Eur. Phys. J. E 3, 323 (2000).

${ }^{29}$ E. R. May, A. Narang, and D. Kopelevich, Phys. Rev. E 76, 021913 (2007).

${ }^{30}$ M. Watson, E. Penev, P. Welch, and F. Brown, J. Chem. Phys. 135, 244701 (2011).

${ }^{31}$ M. Watson, E. Brandt, P. Welch, and F. Brown, Phys. Rev. Lett. 109, 028102 (2012).

${ }^{32}$ E. G. Brandt, A. Braun, J. Sachs, J. Nagle, and O. Edholm, Biophys. J. 100, 2104 (2011).

${ }^{33}$ F. de Meyer, A. Benjamini, J. Rodgers, Y. Misteli, and B. Smit, J. Phys. Chem. B 114, 10451 (2010).

${ }^{34}$ M. Venturoli, M. Sperotto, M. Kranenburg, and B. Smit, Phys. Rep. 437, 1 (2006).

${ }^{35}$ N. Muller, K. Katsov, and M. Schick, Phys. Rep. 434, 113 (2006).

${ }^{36}$ S. Izvekov and G. Voth, J. Phys. Chem. B 109, 2469 (2005).

${ }^{37}$ M. Orsi, D. Haubertin, W. Sanderson, and J. Essex, J. Phys. Chem. B 112, 802 (2008).

${ }^{38}$ S. Marrink, H. Risselada, S. Yefimov, D. Tieleman, and A. de Vries, J. Phys. Chem. B 111, 7812 (2007).

${ }^{39}$ B. Hess, J. Chem. Theory Comput. 4, 435 (2008).

${ }^{40}$ C. Loison, M. Mareschal, K. Kremer, and F. Schmid, J. Chem. Phys. 119, 13138 (2003).

${ }^{41}$ F. Buff, R. A. Lovett, and F. Stillinger, Phys. Rev. Lett. 15, 621 (1965).

${ }^{42}$ R. Evans, Adv. Phys. 28, 143 (1979).

${ }^{43} \mathrm{~J}$. Percus in Fluid Interfacial Phenomena, edited by C. Croxton (John Wiley, New York, 1986), pp. 1-44.

${ }^{44}$ E. Chacón and P. Tarazona, Phys. Rev. Lett. 91, 166103 (2003).

${ }^{45}$ P. Tarazona and E. Chacón, Phys. Rev. B 70, 235407 (2004).

${ }^{46}$ J. Chowdhary and B. M. Ladanyi, J. Phys. Chem. B 110(31), 15442 (2006).

${ }^{47}$ F. Bresme, E. Chacón, P. Tarazona, and K. Tay, Phys. Rev. Lett. 101, 056102 (2008)

${ }^{48}$ L. Partay, G. Hantal, P. Jedlovszky, A. Vincze, and G. Horvai, J. Comput. Chem. 29, 945 (2008).

${ }^{49}$ A. Willard and D. Chandler, J. Phys. Chem. B 114, 1954 (2010).

${ }^{50}$ M. Jorge, P. Jedlovszky, and N. Cordeiro, J. Phys. Chem. C 114, 11169 (2010).

${ }^{51}$ M. Jorge, G. Hantal, P. Jedlovszky, and N. Cordeiro, J. Phys. Chem. C 114, 18656 (2010).

${ }^{52}$ S. Pandit, D. Bostick, and M. Berkowitz, J. Chem. Phys. 119, 2199 (2003).

${ }^{53}$ E. Chacón, E. M. Fernández, D. Duque, R. Delgado-Buscalioni, and P. Tarazona, Phys. Rev. B 80, 195403 (2009).

${ }^{54}$ H. Martinez, E. Chacón, P. Tarazona, and F. Bresme, Proc. R. Soc. A 467, 1939 (2011).

${ }^{55}$ F. Bresme, E. Chacón, H. Martinez, and P. Tarazona, J. Chem. Phys. 134, 214701 (2011).

${ }^{56}$ P. Tarazona, H. Martinez, E. Chacón, and F. Bresme, Phys. Rev. B 85, 085402 (2012).

${ }^{57}$ J. Israelachvili and H. Wennerstrom, J. Phys. Chem. 96, 520 (1992).

${ }^{58}$ See supplementary material at http://dx.doi.org/10.1063/1.4818421 for the analysis of the dependence of the fluctuations spectrum with the approach used to construct $\xi(\mathbf{R})$.

${ }^{59}$ D. Marsh, Chem. Phys. Lipids 144, 146 (2006).

${ }^{60}$ N. Kucerka, S. Tristram-Nagle, and J. Nagle, J. Membr. Biol. 208, 193 (2006). 
${ }^{61}$ H. Bouvrais, P. Méléard, T. Pott, K. Jensen, J. Brask, and J. Ipsen, Biophys. Chem. 137, 7 (2008).

${ }^{62}$ J. Nagel, Faraday Discuss. 161, 11-29 (2013).

${ }^{63}$ L. Fernandez-Puente, I. Bivas, M. Mitov, and P. Meleard, Europhys. Lett. 28, 181 (1994).
${ }^{64}$ S. Marrink, A. de Vries, and A. Mark, J. Phys. Chem. B 108, 750 (2004).

${ }^{65}$ W. den Otter and S. Shkulipa, Biophys. J. 93, 423 (2007).

${ }^{66} \mathrm{~S}$. Shkulipa, Ph.D. thesis, University of Twente, Enschede, The Netherlands, 2006. 